\title{
edoc
}

Institutional Repository of the University of Basel

University Library

Schoenbeinstrasse 18-20

CH-4056 Basel, Switzerland

http://edoc.unibas.ch/

Year: 2014

\section{A uniqueness result for the continuity equation in two dimensions}

Alberti, Giovanni and Bianchini, Stefano and Crippa, Gianluca

Posted at edoc, University of Basel

Official URL: http://edoc.unibas.ch/dok/A6183952

Originally published as:

Alberti, Giovanni and Bianchini, Stefano and Crippa, Gianluca. (2014) A uniqueness result for the continuity equation in two dimensions. Journal of the European Mathematical Society, Vol. 16, H. 2. S. 201234.

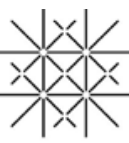




\title{
A uniqueness result for the continuity equation in two dimensions
}

\author{
Giovanni Alberti, Stefano Bianchini, Gianluca Crippa
}

Dedicated to Constantine Dafermos on the occasion of his $70^{\text {th }}$ birthday

\begin{abstract}
We characterize the autonomous, divergence-free vector fields $b$ on the plane such that the Cauchy problem for the continuity equation $\partial_{t} u+\operatorname{div}(b u)=0$ admits a unique bounded solution (in the weak sense) for every bounded initial datum; the characterization is given in terms of a property of Sard type for the potential $f$ associated to $b$. As a corollary we obtain uniqueness under the assumption that the curl of $b$ is a measure. This result can be extended to certain non-autonomous vector fields $b$ with bounded divergence.
\end{abstract}

KEYWORDS: continuity equation, transport equation, uniqueness of weak solutions, weak Sard property, disintegration of measures, coarea formula.

MSC (2010): 35F10, 35L03, 28A50.

\section{INTRODUCTION}

In this paper we consider the continuity equation

$$
\partial_{t} u+\operatorname{div}(b u)=0
$$

where $b:[0, T) \times \mathbb{R}^{d} \rightarrow \mathbb{R}^{d}$ is a bounded, time-dependent vector field on $\mathbb{R}^{d}$ (with $T$ possibly equal to $+\infty$ ). We recall that (1.1) is the equation satisfied by the density $u$ of a continuous distribution of particles moving according to the velocity field $b$, that is, $u(t, x)$ is the number of particles per unit volume at time $t$ and position $x$, and the trajectory of each particle satisfies the ordinary differential equation

$$
\dot{x}=b(t, x) .
$$

Through this paper, the vector field $b$ will not be any more regular than bounded and with bounded (distributional) divergence. 11 Accordingly, solutions of the Cauchy problem for (1.1) are intended in the weak (or distributional) sense: a function $u:[0, T) \times \mathbb{R}^{d} \rightarrow \mathbb{R}$ solves (1.1) with the initial condition

\footnotetext{
${ }^{1}$ In particular $b$ is far from being Lipschitz in the space variable, which is the minimal regularity required to apply the method of characteristics to 1.1.
} 


$$
\begin{aligned}
& u(0, \cdot)=u_{0} \text { if } \\
& \int_{0}^{T} \int_{\mathbb{R}^{d}}\left(\partial_{t} \varphi+b \cdot \nabla \varphi\right) u d x d t+\int_{\mathbb{R}^{d}} \varphi(0, \cdot) u_{0} d x=0 \\
& \forall \varphi \in C_{c}^{\infty}\left([0, T) \times \mathbb{R}^{d}\right) .
\end{aligned}
$$

Concerning the existence of solutions, it can be shown that if the divergence of $b$ is bounded from below then, for every bounded initial datum $u_{0}$, a solution of the Cauchy problem for (1.1) exists for all times in the future and is bounded for finite times $\sqrt[2]{2}$

We focus therefore on the problem of uniqueness, and precisely on the following question: Under which assumption on $b$ does the Cauchy problem for (1.1) admit a unique bounded solution for every bounded initial datum $u_{0}$ ?

In the fundamental paper [13], R.J. DiPerna and P.-L. Lions have proved this uniqueness result under the assumption that $b$ is in the Sobolev class $W^{1,1}$ (locally in space), and later L. Ambrosio 44 generalized this result to vector fields $b$ of class $B V$ (locally in space) $\left.]^{3}\right]^{4}$

When $b$ is a divergence-free autonomous vector field on the plane $(d=2)$, uniqueness has been proved replacing the $B V$ or Sobolev regularity of $b$ by various assumptions on the direction of $b([7,17$, see also [10, 8] for the bounded divergence case). In the opposite direction, N. Depauw [11] gave an example of time-dependent, divergence-free, bounded vector field $b$ on the plane for which there is no uniqueness (see also [1, 9]).

We can now turn to the main result of this paper. Let $b: \mathbb{R}^{2} \rightarrow \mathbb{R}^{2}$ be a bounded, divergence-free, autonomous vector field on the plane; then $b$ admits a Lipschitz potential $f: \mathbb{R}^{2} \rightarrow \mathbb{R}$, that is, $b=\nabla^{\perp} f$, where $\nabla^{\perp}:=\left(-\partial_{2}, \partial_{1}\right)$. In Theorem 4.7 we prove that the Cauchy problem for (1.1) admits a unique bounded solution for every bounded initial datum if and only if the potential $f$ satisfies what we call weak Sard property.

We recall that a differentiable function $f: \mathbb{R}^{2} \rightarrow \mathbb{R}$ satisfies the (strong) Sard property if the image according to $f$ of the critical set $S$ (the set of all $x$ where

\footnotetext{
${ }^{2}$ The bound $\operatorname{div} b \geq-m$ implies that the Jacobian determinant $J$ of the flux associated to 1.2 satisfies $J \geq e^{-m t}$, and therefore, according to the mechanical interpretation given above, the particle density $u$ should satisfy $\|u(t, \cdot)\|_{\infty} \leq e^{m t}\left\|u_{0}\right\|_{\infty}$. This estimate can indeed be proved for smooth $b$, and then used to obtain solutions for non-smooth $b$ by approximation.

${ }^{3}$ Both uniqueness results, besides being deeply interesting per se, had relevant applications to other problems, among which we mention the Boltzmann [14 and Vlasov-Poisson equations [15, and the Keyfitz-Kranzer system [5].

${ }^{4}$ The mechanical interpretation given above suggests that uniqueness for 1.1 should be connected to the uniqueness of solutions of 1.2 with initial condition $x(0)=x_{0}$ for a generic initial point $x_{0}$, but not necessarily for every $x_{0}$. This relation can be made rigorous in terms of uniqueness of the regular Lagrangian flow associated to (1.2) (see 4]), and explains why the regularity assumption on $b$ in the theorems of DiPerna-Lions and Ambrosio are distinctly weaker than those typically required for the uniqueness for 1.2 for every initial point.
} 
$\nabla f(x)=0)$ is negligible, that is, $\mathscr{L}^{1}(f(S))=05^{5}$ The weak Sard property is a measure theoretic version of the Sard property; the precise formulation requires a few additional definitions, and will be given in $\$ 2.13$. Conditions on $f$ (and therefore on $b$ ) that imply the weak Sard property are discussed in detail in 2.15 here we just recall that this property holds whenever $b$ is different than 0 a.e. (2.15(i)), or is of class $B V{ }^{6}$ indeed it suffices that the curl of $b$ is a measure (\$2.15(vi)).

We stress once again that while the results in the literature give only sufficient conditions for uniqueness, Theorem 4.7 gives a necessary and sufficient condition. Accordingly, the examples of functions without the weak Sard property constructed in the companion paper 2] immediately yield examples of divergence-free autonomous vector fields in the plane for which there is no uniqueness (Corollary 4.8). Note that the weak Sard property, like the Sard property, is completely nonlinear in character, and is satisfied by a generic divergence-free vector field (in the category sense, see $\$ 2.15(\mathrm{i})$ ).

We conclude this introduction with an outline of the proof of Theorem 4.7 An essential ingredient is the description of (generic) level sets of Lipschitz functions on the plane given in [2]; we refer to this paper for a detailed discussion of all related measure-theoretic and real analytic issues, and in particular for counterexamples.

The first step is a dimension-reduction argument (Theorem 3.10) that can be summarized as follows: a bounded function $u$ solves the continuity equation (1.1) if and only if it solves a suitable one-dimensional variant of the same equation on every nontrivial connected component of the level set $E_{y}:=f^{-1}(y)$ for almost every $y \mid 7$ In other words, the problem of uniqueness for equation (1.1) is reduced to the uniqueness for the corresponding equations on the nontrivial connected components of a (generic) level set $E_{y}$.

The proof of Theorem 3.10 relies on the notion of disintegration of a measure with respect to the level sets of a function, and on the coarea formula for Lipschitz functions, and holds in every space dimension. The rest of the proof of Theorem 4.7 is strictly two-dimensional.

The next key fact is that for a.e. $y$ every connected component $C$ of $E_{y}$ is a rectifiable simple curve (as shown in [2]), and more precisely it is possible to choose an interval $I$ and a Lipschitz parametrization $\gamma: I \rightarrow C$ so that, under

\footnotetext{
${ }^{5}$ Sard theorem states that functions of class $C^{2}$ have the Sard property (see [20], or [18 Chapter 3, Theorem 1.3]); while this is not always the case for functions of class $C^{1}$.

6 Thus we recover the uniqueness results in [7, 17] in full generality, and those in [13, 4] limited to our particular class of $b$.

7 The notion of "solving the equation on (a subset of) a level set" is properly defined in 33.6 a connected component is nontrivial if it contains more than one point. Note that this statement is a close relative of the method of characteristics: indeed the vector field $b$, being orthogonal to $\nabla f$, is tangent to the level set $E_{y}$ at a.e. point (with respect to the length measure) and for a.e. $y$. Hence these level sets are the proper replacement for characteristic curves.
} 
the change of variable $x=\gamma(s)$, the equation on $C$ becomes

$$
\partial_{t}(u(1+\lambda))+\partial_{s} u=0,
$$

where $\lambda$ is a suitable singular measure on $I]^{8}$ Note that, due to the particular choice of $\gamma$, the vector field $b$ no longer appears in the equation.

Next we note that the Cauchy problem for (1.4) admits a unique bounded solution for every bounded initial datum if and only if the measure $\lambda$ is trivial. Indeed, if $\lambda=0$ equation (1.4) reduces to $\partial_{t} u+\partial_{s} u=0$, for which uniqueness is well-known. To understand why the converse holds, assume that $I$ is the real line and $\lambda$ is a Dirac mass at 0 ; if $u$ represents the density of a distribution of particles, then equation (1.4) means that each particle moves at constant speed 1 from left to right, except when it reaches the point 0 , where it may stop for any given amount of time. Therefore, if $u_{0}$ is an initial datum with support contained in $(-\infty, 0)$, a solution of $(1.4)$ with initial condition $u(0, s)=u_{0}(s)$ is the function $u:[0,+\infty) \times \mathbb{R} \rightarrow \mathbb{R}$ defined by

$$
u(t, s):= \begin{cases}u_{0}(s-t) & \text { if } s \neq 0 \\ 0 & \text { if } s=0\end{cases}
$$

(no particle stops at 0), while another solution is

$$
\tilde{u}(t, s):= \begin{cases}u_{0}(s-t) & \text { if } s<0 \\ \int_{-t}^{0} u_{0}(\tau) d \tau & \text { if } s=0 \\ 0 & \text { if } s>0\end{cases}
$$

(all particles stop at 0).

We conclude that uniqueness for (1.1) holds if and only if $\lambda=0$ for every nontrivial connected component $C$ of a.e. level set $E_{y}$, which in turn is equivalent to the weak Sard property (Lemma 2.14). Without entering into details, we just mention that the connection between the weak Sard property, which is related to the critical set $S$, and the measures $\lambda$ lies in the fact that these measures are given by the disintegration (with respect to the level sets of $f$ ) of the restriction of Lebesgue measure to $S \cap E^{*}$, where $E^{*}$ is the union of all nontrivial connected components of all levels sets of $f$.

As one might expect, Theorem 4.7 can be extended in many different ways. In Theorem 5.2 we consider the case of a vector field on the plane of the form $b=a \nabla^{\perp} f$, where $f$ is a Lipschitz function as above and $a$ a scalar function depending also on time. Further extensions are mentioned in $\$ 6.1$. It is indeed conceivable to apply the strategy outlined above even in higher space dimension (see $\$ 6.2$ ).

This paper is organized as follows: Section 2 contains the measure-theoretic and real-analytica results used in the rest of the paper: the disintegration of a measure with respect to the level sets of a function, the coarea formula for

\footnotetext{
${ }^{8}$ The precise definition of $\lambda$ and the weak formulation of 1.4 are given in Lemma 4.4
} 
Lipschitz functions, the description of connected components of the level sets of a Lipschitz function on the plane, the definition of weak Sard property and some related results. Section 3 is devoted to the dimension-reduction argument (Theorem 3.10); Section 4 and Section 5 contain the proof of the main result (Theorem 4.7) and of the generalization mentioned above (Theorem 5.2). Section 6 contains some additional remarks, and finally the Appendix contains a measurable selection lemma used in the proof of Theorem 3.10 .

Acknowledgements. This work has been partially supported by the italian Ministry of Education, University and Research (MIUR) through the 2008 PRIN Grant "Trasporto ottimo di massa, disuguaglianze geometriche e funzionali e applicazioni", and by the European Research Council through the 2009 Starting Grant "Hyperbolic Systems of Conservation Laws: singular limits, properties of solutions and control problems". During the completion of this paper the first author was visiting SISSA in Trieste, whose support and hospitality he gratefully acknowledges.

\section{Measure theoretic Preliminaries}

We begin this section by recalling the notion of disintegration of a measure, focusing in particular on the disintegration of the Lebesgue measure with respect to Lipschitz maps ( 2.7 and Lemma 2.8). We then restrict our attention to Lipschitz functions on the plane (Lemma 2.11); in this setting we introduce the notion of weak Sard property ( $\$ 2.13$ ), prove a characterization of this property in terms of disintegration of the Lebesgue measure (Lemma 2.14), and list some conditions which imply it (2.15). We conclude with a change-of-variable formula used in the proof of Lemma 4.5 .

2.1. Basic notation. In this paper we follow the standard notation of measure theory. Sets and functions are always assumed to be Borel measurable, and measures are always defined on the Borel $\sigma$-algebra of some locally compact, separable metric space $X$. Unless otherwise stated, a measure on $X$ is assumed to be positive and locally finite. We write $\lambda \ll \mu$ when the measure $\lambda$ is absolutely continuous with respect to the measure $\mu$, and $\lambda \perp \mu$ when $\lambda$ is singular with respect to $\mu 9^{9}$ We say that the measure $\mu$ is supported on the set $E$ if $\mu(X \backslash E)=0.10$

Given a function $\rho: X \rightarrow[0,+\infty]$, we denote by $\rho \cdot \mu$ the measure on $X$ defined by

$$
(\rho \cdot \mu)(E):=\int_{E} \rho d \mu
$$

\footnotetext{
${ }^{9}$ Recall that $\lambda \ll \mu$ if $\lambda(E)=0$ whenever $\mu(E)=0$, or equivalently (in the context of this paper) when $\lambda$ can be written as $\lambda=\rho \cdot \mu$ for a suitable density $\rho$; $\lambda$ is singular with respect to $\mu$ if it is supported on a $\mu$-negligible set, or equivalently if $\lambda$ and $\mu$ are supported on disjoint sets.

10 Note that $E$ does not need to be closed, and does not necessarily contain the support of $\mu$ (defined in the usual distributional sense).
} 
for every Borel set $E$ contained in $X$. Given a set $A$ contained in $X$, we write $\mathbf{1}_{A}: X \rightarrow\{0,1\}$ for the characteristic function of $A$, and therefore $\mathbf{1}_{A} \cdot \mu$ is the restriction of $\mu$ to $A$.

Given a metric space $Y$ and a map $f: X \rightarrow Y$, the push-forward of $\mu$ according to $f$ is the measure $f_{\#} \mu$ on $Y$ defined by

$$
\left[f_{\#} \mu\right](E):=\mu\left(f^{-1}(E)\right)
$$

for every Borel set $E$ contained in $Y$. Thus

$$
\int_{Y} \varphi d\left(f_{\#} \mu\right)=\int_{X} \varphi \circ f d \mu
$$

for every function $\varphi: Y \rightarrow[0,+\infty]$, and therefore also for every possibly vectorvalued $\varphi$ in $L^{1}\left(f_{\#} \mu\right)$.

As usual, $\mathscr{L}^{d}$ is the Lebesgue measure on (domains in) $\mathbb{R}^{d}$ while $\mathscr{H}^{d}$ is the $d$ dimensional Hausdorff measure on every metric space - the usual $d$-dimensional volume for $d$-dimensional surfaces of class $C^{1}$ in some Euclidean space. When the measure is not specified, it is assumed to be the Lebesgue measure; along this line we often write $\int g(x) d x$ instead of $\int g d \mathscr{L}^{d}$.

2.2. Borel families of measures. Let $Y$ be a metric space and $\left\{\mu_{y}: y \in Y\right\}$ a family of measures on a locally compact, separable metric space $X$. We say that such family, or more precisely the map $y \mapsto \mu_{y}$, is Borel if the function

$$
y \mapsto \int_{X} \varphi d \mu_{y}
$$

is Borel for every test function $\varphi: X \rightarrow \mathbb{R}$ which is continuous and compactly supported ${ }^{11}$ It then follows that the function in 2.1 is Borel measurable also for every Borel test function $\varphi: X \times Y \rightarrow[-\infty,+\infty]$ which is either positive or such that $\varphi(\cdot, y)$ belongs to $L^{1}\left(\mu_{y}\right)$ for every $y$.

2.3. Disintegration of measures. Let $X$ and $Y$ be locally compact, separable metric spaces, $\mu$ a measure on $X, f: X \rightarrow Y$ a Borel map, and $\nu$ a measure on $Y$ such that $f_{\#} \mu \ll \nu$. Then there exists a Borel family $\left\{\mu_{y}: y \in Y\right\}$ of measures on $X$ such that

(i) $\mu_{y}$ is supported on the level set $E_{y}:=f^{-1}(y)$ for every $y \in Y$;

(ii) $\mu$ can be decomposed as $\mu=\int_{Y} \mu_{y} d \nu(y)$, which means that

$$
\mu(A)=\int_{Y} \mu_{y}(A) d \nu(y)
$$

for every Borel set $A$ contained in $X$.

Any family $\left\{\mu_{y}\right\}$ satisfying (i) and (ii) is called a disintegration of $\mu$ with respect to $f$ and $\nu$. The disintegration is unique in the following sense: for any other disintegration $\left\{\tilde{\mu}_{y}\right\}$ there holds $\mu_{y}=\tilde{\mu}_{y}$ for $\nu$-a.e. $y \in Y$.

\footnotetext{
11 This is equivalent to the notion of Borel measurability for maps with values in the space of locally finite measures on $X$, when the latter has been endowed with the weak* topology as dual of the space of continuous functions with compact support.
} 
The existence and uniqueness of the disintegration is a standard result in case $X$ is compact, $\mu$ is finite, and $\nu:=f_{\#} \mu$ (see for instance [12, Chapter III, $\S 70$ and $\S 71]$ ). The statement given above can be easily derived from this particular case.

2.4. Properties of disintegration. We list here some general properties of the disintegration that will be used (often tacitly) through the paper.

(i) Formula 2.2$)$ implies that

$$
\int_{X} \varphi d \mu=\int_{Y}\left[\int_{E_{y}} \varphi d \mu_{y}\right] d \nu(y)
$$

for every Borel function $\varphi: X \rightarrow[0,+\infty]$.

If $\varphi$ is a real- or vector-valued function in $L^{1}(\mu)$, by applying identity (2.3) with $|\varphi|$ in place of $\varphi$ we obtain that $\varphi$ belongs to $L^{1}\left(\mu_{y}\right)$ for $\nu$-a.e. $y$, and the function $y \mapsto \int|\varphi| d \mu_{y}$ is in $L^{1}(\nu)$. Thus both sides of (2.3) make sense, and the equality holds even for such $\varphi$.

(ii) If $A$ is a set in $X$ with $\mu(A)<+\infty$, formula $(2.2)$ implies that $\mu_{y}(A)$ is finite for $\nu$-a.e. $y$. In particular, if $\mu$ is finite then $\mu_{y}$ is finite for $\nu$-a.e. $y$.

(iii) Formula (2.2) shows that a set $A$ in $X$ is $\mu$-negligible if and only if it is $\mu_{y}$-negligible for $\nu$-a.e. $y$. We infer the following: (a) $\mu\left(f^{-1}(N)\right)=0$ for every set $N$ such that $\nu(N)=0$; (b) $\mu$ is supported on a set $F$ if and only if $\mu_{y}$ is supported on $F \cap E_{y}$ for $\nu$-a.e. $y$; (c) if $P(x)$ is a proposition that depends on the variable $x \in X$, then $P(x)$ holds for $\mu$-a.e. $x$ if and only if it holds for $\mu_{y}$-a.e. $x$ and $\nu$-a.e. $y$.

(iv) If $\mu^{\prime}$ is a measure on the metric space $X^{\prime}$, we write $\mu \otimes \mu^{\prime}$ for the product measure on $X \times X^{\prime}$ and we consider the function $\tilde{f}: X \times X^{\prime} \rightarrow Y$ defined by $\tilde{f}\left(x, x^{\prime}\right)=f(x)$. Then, the disintegration of $\mu \otimes \mu^{\prime}$ with respect to $\tilde{f}$ and $\nu$ is $\left\{\mu_{y} \otimes \mu^{\prime}\right\}$.

2.5. Lipschitz maps. For the rest of this section $d, k$ are integers such that $0 \leq k<d$, and $f: \mathbb{R}^{d} \rightarrow \mathbb{R}^{d-k}$ is a Lipschitz map.

For every $y \in \mathbb{R}^{d-k}$ we write $E_{y}$ for the level set $f^{-1}(y)$.

By Rademacher theorem $f$ is differentiable at almost every point in $\mathbb{R}^{d}$, and in all such points we define the Jacobian

$$
J:=\left[\operatorname{det}\left(\nabla f \cdot \nabla^{t} f\right)\right]^{1 / 2} .
$$

We call critical set of $f$ the set $S$ of all points in $\mathbb{R}^{d}$ where either $f$ is not differentiable or $J=0$, that is, the rank of $\nabla f$ is strictly less than $d-k$.

A connected component $C$ of $E_{y}$ is a connected subset of $E_{y}$ which is maximal with respect to inclusion. Notice that every such connected component is a closed set.

For every $y \in \mathbb{R}^{d-k}$ we denote by $\mathscr{C}_{y}$ the family of all connected components $C$ of $E_{y}$ such that $\mathscr{H}^{k}(C)>0$; we then define $E_{y}^{*}$ as the union of all $C$ in $\mathscr{C}_{y}$, and $E^{*}$ as the union of all $E_{y}^{*}$ with $y \in \mathbb{R}^{d-k}$. Note that the sets $E^{*}$ and $E_{y}^{*}$ are Borel (see [2, Proposition 6.1]). 
2.6. Lemma. In the context of the previous paragraph, the following statements hold:

(i) if $\mu:=J \cdot \mathscr{L}^{d}$ then $f_{\#} \mu$ is absolutely continuous with respect to $\mathscr{L}^{d-k}$ and its disintegration with respect to $f$ and $\mathscr{L}^{d-k}$ is $\mu_{y}:=\mathbf{1}_{E_{y}} \cdot \mathscr{H}^{k}$;

(ii) for a.e. $y \in \mathbb{R}^{d-k}$ there holds $\mathscr{H}^{k}\left(E_{y} \cap S\right)=0$, and for every bounded set $A$ in $\mathbb{R}^{d}$ we have $\mathscr{H}^{k}\left(A \cap E_{y}\right)<+\infty$ and

$$
\int_{A \cap E_{y}} \frac{1}{J} d \mathscr{H}^{k}<+\infty
$$

(iii) if $\tilde{\mu}:=\mathbf{1}_{\mathbb{R}^{d} \backslash S} \cdot \mathscr{L}^{d}$ then $f_{\#} \tilde{\mu}$ is absolutely continuous with respect to $\mathscr{L}^{d-k}$, and its disintegration with respect to $f$ and $\mathscr{L}^{d-k}$ is given by $\tilde{\mu}_{y}:=(1 / J) \mathbf{1}_{E_{y}} \cdot \mathscr{H}^{k}$;

(iv) the family $\mathscr{C}_{y}$ is countable and $\mathscr{H}^{k}\left(E_{y} \backslash E_{y}^{*}\right)=0$ for $\mathscr{L}^{d-k}$-a.e. $y$;

(v) $\mathscr{L}^{d}\left(\mathbb{R}^{d} \backslash\left(E^{*} \cup S\right)\right)=0$.

Proof. Statement (i) is just a reformulation of the coarea formula for Lipschitz maps, see for instance [16, §3.2.11], [21, §10], or [19, Corollary 5.2.6].

Using statement (i) and formula $(2.2)$ we obtain

$$
0=\mu(S)=\int_{\mathbb{R}^{d-k}} \mu_{y}(S) d y=\int_{\mathbb{R}^{d-k}} \mathscr{H}^{k}\left(E_{y} \cap S\right) d y,
$$

and therefore $\mathscr{H}^{k}\left(E_{y} \cap S\right)=0$ for a.e. $y$. Similarly,

$$
+\infty>\mu(A)=\int_{\mathbb{R}^{d-k}} \mu_{y}(A) d y=\int_{\mathbb{R}^{d-k}} \mathscr{H}^{k}\left(A \cap E_{y}\right) d y
$$

implies that $\mathscr{H}^{k}\left(E_{y} \cap A\right)$ is finite for a.e. $y$. The last part of the statement follows by applying 2.3 with $\varphi:=(1 / J) \mathbf{1}_{A \backslash S}$ :

$$
\begin{aligned}
+\infty>\mathscr{L}^{d}(A \backslash S)=\int \varphi d \mu & =\int_{\mathbb{R}^{d-k}}\left[\int_{E_{y}} \varphi d \mu_{y}\right] d y \\
& =\int_{\mathbb{R}^{d-k}}\left[\int_{A \cap E_{y}} \frac{1}{J} d \mathscr{H}^{k}\right] d y .
\end{aligned}
$$

Similarly, statement (iii) follows by applying 2.3) with $\varphi:=(1 / J) \mathbf{1}_{A \backslash S}$ and $A$ an arbitrary Borel set in $\mathbb{R}^{d}$.

Statement (iv) is proved in [2, Theorem 2.5(iii)].

Statement (v) follows from statements (iii) and (iv):

$$
\begin{aligned}
\mathscr{L}^{d}\left(\mathbb{R}^{d} \backslash\left(E^{*} \cup S\right)\right)=\tilde{\mu}\left(\mathbb{R}^{d} \backslash E^{*}\right) & =\int_{\mathbb{R}^{d-k}} \tilde{\mu}_{y}\left(\mathbb{R}^{d} \backslash E^{*}\right) d y \\
& =\int_{\mathbb{R}^{d-k}}\left[\int_{E_{y} \backslash E_{y}^{*}} \frac{1}{J} d \mathscr{H}^{k}\right] d y=0 .
\end{aligned}
$$


2.7. Disintegration of Lebesgue measure. We take $f$ as above and choose a measure $\nu^{s}$ on $\mathbb{R}^{d-k}$ so that $\nu^{s}$ is singular with respect to $\mathscr{L}^{d-k}$ and $f_{\#} \mathscr{L}^{d} \ll \nu$ with $\nu:=\mathscr{L}^{d-k}+\nu^{s}$. For the rest of this section we denote by $\left\{\mu_{y}\right\}$ the disintegration of $\mathscr{L}^{d}$ with respect to $f$ and $\nu$.

2.8. Lemma. In the context of the previous paragraph, the following statements hold:

(i) for $\nu^{s}$-a.e. $y$ the measure $\mu_{y}$ is supported on $S \cap E_{y}$;

(ii) for $\mathscr{L}^{d-k}$-a.e. $y$ the measure $\mu_{y}$ can be decomposed as

$$
\mu_{y}=(1 / J) \mathbf{1}_{E_{y} \backslash S} \cdot \mathscr{H}^{k}+\mu_{y}^{s}
$$

with $\mu_{y}^{s}$ a measure supported on $E_{y} \cap S$; moreover $\mu_{y}^{s}$ is singular with respect to $(1 / J) \mathbf{1}_{E_{y} \backslash S} \cdot \mathscr{H}^{k}$, and the latter measure agrees with $(1 / J) \mathbf{1}_{E_{y}} \cdot \mathscr{H}^{k}$.

Proof. Note that $\mathscr{L}^{d}$ can be written as the sum of $\mu^{1}:=\mathbf{1}_{S} \cdot \mathscr{L}^{d}$ and $\mu^{2}:=$ $\mathbf{1}_{\mathbb{R}^{d} \backslash S} \cdot \mathscr{L}^{d}$, and therefore its disintegration (with respect to $f$ and $\nu$ ) is obtained by summing the disintegrations of $\mu^{1}$ and $\mu^{2}$.

Now $\mu^{1}$ is supported on $S$ and then $\mu_{y}^{1}$ is supported on $S \cap E_{y}$ for $\nu$-a.e. $y$; on the other hand, Lemma 2.6(iii) implies that $\mu_{y}^{2}=0$ for $\nu^{s}$-a.e. $y$ and $\mu_{y}^{2}=$ $\left(\mathbf{1}_{E_{y}} / J\right) \cdot \mathscr{H}^{k}$ for $\mathscr{L}^{d-k}$-a.e. $y$. Putting together these facts we immediately obtain statement (i) and the first part of (ii).

We have that $\mu_{y}^{s}$ is singular with respect to $\left(\mathbf{1}_{E_{y} \backslash S} / J\right) \cdot \mathscr{H}^{k}$ because these measures are supported on the disjoint sets $E_{y} \backslash S$ and $E_{y} \cap S$. The latter measure agrees with $\left(\mathbf{1}_{E_{y}} / J\right) \cdot \mathscr{H}^{k}$ because $\mathscr{H}^{k}\left(E_{y} \cap S\right)=0$ (Lemma 2.6(ii)).

2.9. Lipschitz functions on the plane. For the rest of this section we assume $d=2$ and $k=1$, that is, $f$ is a Lipschitz function on $\mathbb{R}^{2}$. Therefore $J=|\nabla f|$, $S$ is the set of all points where either $f$ is not differentiable or $\nabla f=0$, and $\mathscr{C}_{y}$ is the family of all nontrivial connected components of the level set $E_{y}$, namely those which contain more than one point.

2.10. Simple curves. We denote by $[0, L]^{*}$ the space obtained by identifying the endpoints of the interval $[0, L]$, endowed with the distance

$$
d(x, y):=|x-y| \wedge(L-|x-y|) .
$$

The canonical measure on $[0, L]^{*}$ is the Lebesgue measure, which we denote simply by $\mathscr{L}$.

We say that a set $C$ in $\mathbb{R}^{d}$ is a closed, simple curve with finite length if there exist $L>0$ and a Lipschitz bijection $\gamma:[0, L]^{*} \rightarrow C$. We call any such $\gamma$ a parametrization of $C$.

2.11. Lemma. Assume that $f$ has compact support, and take $\mu_{y}, \mu_{y}^{s}$ as in Lemma 2.8. The following statements hold for $\mathscr{L}^{1}$-a.e. $y \in \mathbb{R}$ and every connected component $C \in \mathscr{C}_{y}$ :

(i) $C$ is a closed simple curve with finite length; 
(ii) there exists a parametrization $\gamma:[0, L]^{*} \rightarrow C$ such that $\dot{\gamma}=\nabla^{\perp} f(\gamma) \neq$ 0 a.e. (recall that $\left.\nabla^{\perp}:=\left(-\partial_{2}, \partial_{1}\right)\right)$;

(iii) the push-forward of $\mathscr{L}$ according to $\gamma$ is $(1 / J) \mathbf{1}_{C} \cdot \mathscr{H}^{1}$;

(iv) there exists a measure $\lambda$ on $[0, L]^{*}$ such that $\lambda$ is singular with respect to $\mathscr{L}$, and the push-forward of $\lambda$ according to $\gamma$ is the restriction of $\mu_{y}^{s}$ to $C$;

(v) the push-forward of $\mathscr{L}+\lambda$ according to $\gamma$ is the restriction of $\mu_{y}$ to $C$.

2.12. Remark. (i) The assumption that $f$ has compact support was made for the sake of simplicity, and it can be easily removed. In that case statement (i) should be modified, allowing for connected components that are simple curves with end points at infinity.

(ii) It is always possible to choose the parametrization $\gamma$ in such a way that the measure $\lambda$ has no atom at 0 , that is, $\lambda(\{0\})=0$. This assumption makes certain proofs much simpler, and will be always tacitly made in the following.

Proof. By Lemma 2.8(ii) we can assume that $\mathscr{H}^{1}(S \cap C)=0$ and

$$
\int_{C} \frac{1}{J} d \mathscr{H}^{1}<+\infty \text {. }
$$

Statement (i) is proved in [2, Theorem 2.5(iv)].

The same theorem shows that $C$ admits a parametrization $\varphi:\left[0, L^{\prime}\right]^{*} \rightarrow C$ such that

$$
J(\varphi) \neq 0 \quad \text { and } \quad \dot{\varphi}=\frac{\nabla^{\perp} f(\varphi)}{|\nabla f(\varphi)|}=\frac{\nabla^{\perp} f(\varphi)}{J(\varphi)} \text { a.e. }
$$

Thus the parametrization in statement (ii) is given by $\gamma:=\varphi \circ \tau^{-1}$ where $\tau:\left[0, L^{\prime}\right] \rightarrow[0, L]$ is the function defined by

$$
\tau(t):=\int_{0}^{t} \frac{1}{J(\varphi(s))} d s \quad \text { and } \quad L:=\int_{0}^{L^{\prime}} \frac{1}{J(\varphi(s))} d s .
$$

The definition of $\tau$ is well-posed because the integral that defines $L$ is finite, and more precisely it agrees with the integral at the left-hand side of (2.4) (because $|\dot{\varphi}|=1$ a.e.). Since $J \leq m$, where $m$ denotes the Lipschitz constant of $f$, we have that

$$
\tau\left(t_{2}\right)-\tau\left(t_{1}\right) \geq \frac{1}{m}\left(t_{2}-t_{1}\right)
$$

whenever $t_{1}<t_{2}$, which implies that $\tau^{-1}$ is a Lipschitz function, and therefore so is $\gamma$. Moreover $\tau$ is of class $W^{1,1}$ and $\dot{\tau}=1 / J(\varphi) \neq 0$ a.e.; using this fact one obtains that

$$
\left(\tau^{-1}\right)^{\prime}=J(\gamma)=|\nabla f(\gamma)| \text { a.e. }
$$

This equation and (2.5) yield $\dot{\gamma}=\nabla^{\perp} f(\gamma)$ a.e., concluding the proof of statement (ii).

Statement (iii) follows by the fact that $|\dot{\gamma}|=|\nabla f(\gamma)|=J(\gamma)$ a.e.

To prove statement (iv), let $\lambda$ be the push-forward according to $\gamma^{-1}$ of $\mathbf{1}_{C} \cdot \mu_{y}^{s}$. In order to prove that $\lambda \perp \mathscr{L}$, note that $\mu_{y}^{s}$ is supported on $S \cap C$, and therefore 
$\lambda$ is supported on $\gamma^{-1}(S \cap C)$. Moreover $S \cap C$ is $\mathscr{H}^{1}$-negligible, and therefore $\gamma^{-1}(S \cap C)=\tau\left(\varphi^{-1}(S \cap C)\right)$ is $\mathscr{L}$-negligible because both $\varphi^{-1}$ and $\tau$ maps negligible sets in negligible sets.

Statement (v) follows from statements (iii) and (iv).

2.13. The weak Sard property. Let $f: \mathbb{R}^{2} \rightarrow \mathbb{R}$ be a Lipschitz function $\sqrt{12}$ We say that $f$ has the weak Sard property if the push-forward according to $f$ of the restriction of the Lebesgue measure to $S \cap E^{*}$ (where $S$ and $E^{*}$ have been defined in 2.5 is singular, that is

$$
f_{\#}\left(\mathbf{1}_{S \cap E^{*}} \cdot \mathscr{L}^{2}\right) \perp \mathscr{L}^{1} .
$$

2.14. Lemma. The following statements are equivalent:

(a) $f$ satisfies the weak Sard property;

(b) $\mu_{y}^{s}\left(E_{y}^{*}\right)=0$ for $\mathscr{L}^{1}$-a.e. $y \in \mathbb{R}$;

(c) $\mu_{y}^{s}(C)=0$ for $\mathscr{L}^{1}$-a.e. $y \in \mathbb{R}$ and every $C \in \mathscr{C}_{y}$.

Proof. Take $\nu$ as in 2.7 and $\mu_{y}^{s}$ as Lemma 2.8. Set $\tilde{\mu}:=\mathbf{1}_{S \cap E^{*}} \cdot \mathscr{L}^{2}$, and let $\left\{\tilde{\mu}_{y}\right\}$ be the disintegration of $\tilde{\mu}$ with respect to $f$ and $\nu$. Thus (2.6) can be restated as

$$
\tilde{\mu}_{y}=0 \text { for } \mathscr{L}^{1} \text {-a.e. } y \text {. }
$$

On the other hand Lemma 2.8 (ii) implies that $\tilde{\mu}_{y}=\mathbf{1}_{S \cap E^{*}} \cdot \mu_{y}^{s}$ for $\mathscr{L}^{1}$-a.e. $y$, and therefore $\tilde{\mu}_{y}=0$ if and only if $0=\mu_{y}^{s}\left(S \cap E^{*}\right)=\mu_{y}^{s}\left(E_{y}^{*}\right)$ (for the second equality use that $\mu_{y}^{s}$ is supported on $E_{y} \cap S$ and $\left.E_{y} \cap E^{*}=E_{y}^{*}\right)$.

We have thus proved that 2.6 holds if and only if $\mu_{y}^{s}\left(E_{y}^{*}\right)=0$ for $\mathscr{L}^{1}$-a.e. $y$, that is, statements (a) and (b) are equivalent.

The equivalence of (b) and (c) is immediate because $E_{y}^{*}$ is the union of all $C \in \mathscr{C}_{y}$, and $\mathscr{C}_{y}$ is countable for a.e. $y$ (Lemma 2.6(iv)).

2.15. About the weak Sard property. We list here some conditions on $f$ which imply the weak Sard property. Indeed all of them imply the slightly stronger property

$$
f_{\#}\left(\mathbf{1}_{S} \cdot \mathscr{L}^{2}\right) \perp \mathscr{L}^{1}
$$

Since in Theorem 4.7 the function $f$ is the potential associated to a given vector field $b$ by the relation $\nabla^{\perp} f=b$, we will express the following conditions also in terms of $b$.

(i) The weak Sard property is implied by the condition $\mathscr{L}^{2}\left(S \cap E^{*}\right)=0$, which in turn is implied by $\nabla f \neq 0$ a.e., or, equivalently, $b \neq 0$ a.e. Note that the set of all vector fields $b$ that verify the last condition is residual ${ }^{13}$ in the Banach space $X$ of divergence-free, bounded vector fields endowed with the $L^{\infty}$

\footnotetext{
12 The weak Sard property can be defined in the more general context of Lipschitz maps from $\mathbb{R}^{d}$ into $\mathbb{R}^{d-k}$, but only the case $d=2, k=1$ is relevant to this paper.

${ }^{13} \mathrm{~A}$ set in a topological space is residual if it contains a countable intersection of open dense sets.
} 
norm (this is a reformulation of [2, Proposition 4.10]). In particular the set of all $b$ whose potential $f$ has the weak Sard property is residual in $X$.

(ii) The (strong) Sard property implies the weak Sard property. Indeed the measure $f_{\#}\left(\mathbf{1}_{S \cap E^{*}} \cdot \mathscr{L}^{2}\right)$ is supported on the set $f(S)$, and if this set is negligible (Sard property), then the measure must be singular (weak Sard property).

(iii) A function $f$ on $\mathbb{R}^{2}$ is said to have the Lusin property of order $k$ if there exists a sequence of functions $f_{n}$ of class $C^{k}$ on $\mathbb{R}^{2}$ and Borel sets $A_{n}$ such that $f=f_{n}$ on $A_{n}$, and the sets $A_{n}$ cover almost all of $\mathbb{R}^{2}{ }^{14}$ A Lipschitz function with the Lusin property of order 2 satisfies (2.7) and therefore has the weak Sard property.

Let indeed $S_{n}$ be the critical set of $f_{n}$. Then $S \cap A_{n}$ is contained in $S_{n} \cap A_{n}$ up to a negligible set, and $S$ is contained in the union of $S_{n} \cap A_{n}$ up to a negligible set. Hence the measure $\mathbf{1}_{S \cap E^{*}} \cdot \mathscr{L}^{2}$ is supported on the union of $S_{n} \cap A_{n}$, and its push-forward according to $f$ is supported on the union of all $f\left(S_{n} \cap A_{n}\right)=f_{n}\left(S_{n} \cap A_{n}\right)$, which is negligible because it is contained in the union of the negligible sets $f_{n}\left(S_{n}\right)$.

(iv) Following [23, §3.5.5] we say that $f$ admits an $L^{1}$-Taylor expansion of order 2 at $x$ if $f(x+h)=P_{x}(h)+R_{x}(h)$ for every $h$, where $P_{x}$ is a polynomial of degree 2 , and the remainder $R_{x}$ satisfies

$$
f_{B(r)}\left|R_{x}(h)\right| d h=o\left(r^{2}\right)
$$

(the barred integral stands for the average over the ball $B(r)$ with center 0 and radius $r$ ). If $f$ admits an $L^{1}$-Taylor expansion of order 2 at a.e. point, then it has the Lusin property of order 2 (use the $L^{p}$-version of Whitney extension theorem given in [23, Theorem 3.6.3]), and therefore also the weak Sard property.

(v) If $f$ is locally in the Sobolev class $W^{2,1}$ (or, equivalently, $b$ is locally in the class $\left.W^{1,1}\right)$ then $f$ admits an $L^{1}$-Taylor expansion of order 2 at a.e. point [23, Theorem 3.4.2], and therefore it has the weak Sard property.

(vi) The proof of [23, Theorem 3.4.2] can be easily modified to show that $f$ admits an $L^{1}$-Taylor expansion of order 2 at a.e. point-which implies the weak Sard property - even when the second order distributional derivative of $f$ is locally a vector-valued measure (that is, when $b$ is locally of class $B V$ ). In [3. Proposition 4.4] we prove that the same conclusion holds under the weaker assumption that the distributional Laplacian of $f$ (that is, the curl of $b$ ) is locally a signed measure.

(vii) In [2, Section 4] we construct a function on the plane without the weak Sard property and of class $C^{1, \alpha}$ for every $\alpha<1$.

We conclude this section with a particular change of variable that will be used in the proof of Lemma 4.5 .

2.16. A particular change of variable. In what follows $I$ is the interval $[0, L], \mathscr{L}$ is the Lebesgue measure on $I, \lambda$ is a measure on $I$ which is singular

\footnotetext{
14 Thus Lusin's theorem states that a Borel function has the Lusin property of order 0.
} 
with respect to $\mathscr{L}$, and $\mathscr{A}$ is the set of atoms of $\lambda$ (points with positive measure). We set

and denote by $\widehat{\mathscr{L}}$ the Lebesgue measure on $\widehat{I}$.

$$
\widehat{L}:=(\mathscr{L}+\lambda)(I) \quad \text { and } \quad \widehat{I}:=[0, \widehat{L}],
$$

We denote by $\hat{\sigma}$ the multi-valued function from $I$ to $\widehat{I}$ that to every $s \in I$ associates the interval

$$
\hat{\sigma}(s):=\left[\sigma_{-}(s), \sigma_{+}(s)\right]
$$

where

$$
\hat{\sigma}_{-}(s):=(\mathscr{L}+\lambda)([0, s)) \text { and } \hat{\sigma}_{+}(s):=(\mathscr{L}+\lambda)([0, s]),
$$

and define $\sigma: \widehat{I} \rightarrow I$ as the inverse of $\hat{\sigma}$.

Then $\hat{\sigma}$ is surjective on $\widehat{I}$, strictly increasing, and uni-valued for every $s \notin \mathscr{A}$ because $\hat{\sigma}_{-}$and $\hat{\sigma}_{+}$are strictly increasing, and $\hat{\sigma}_{-}(s)=\hat{\sigma}_{+}(s)$ whenever $s \notin \mathscr{A}$. Moreover

$$
s_{2}-s_{1} \leq \hat{s}_{2}-\hat{s}_{1}
$$

for every $s_{1}, s_{2} \in I$ with $s_{1}<s_{2}$, and every $\hat{s}_{1} \in \hat{\sigma}\left(s_{1}\right), \hat{s}_{2} \in \hat{\sigma}\left(s_{2}\right)$.

Accordingly, $\sigma$ is surjective from $\widehat{I}$ onto $I$, uni-valued and 1-Lipschitz because of (2.8), constant on the interval $\sigma(s)$ for every $s \in \mathscr{A}$ and strictly increasing at every point outside $\sigma(\mathscr{A})$.

2.17. Lemma. Let $F$ be an $\mathscr{L}$-negligible set in $I$ which supports the measure $\lambda$, and set $\widehat{F}:=\hat{\sigma}(F)$. Then

(i) the push-forward of the measure $\widehat{\mathscr{L}}$ according to $\sigma$ is $\mathscr{L}+\lambda$, and this means that for every function $g: I \rightarrow[-\infty,+\infty]$ which is either positive or belongs to $L^{1}(\mathscr{L}+\lambda)$ there holds

$$
\int_{I} g d(\mathscr{L}+\lambda)=\int_{\widehat{I}} g \circ \sigma d \widehat{\mathscr{L}}
$$

(ii) the derivative of $\sigma$ agrees with $\mathbf{1}_{\widehat{I} \backslash \widehat{F}}$ a.e. in $\widehat{I}$.

Proof. Let $E:=\left(s_{1}, s_{2}\right)$ be an open interval in $I$. By the definition of $\sigma$ and $\hat{\sigma}$ we have that

$$
\sigma^{-1}(E)=\hat{\sigma}(E)=\left(\sigma_{+}\left(s_{1}\right), \sigma_{-}\left(s_{2}\right)\right)
$$

and therefore

$$
\widehat{\mathscr{L}}\left(\sigma^{-1}(E)\right)=\sigma_{-}\left(s_{2}\right)-\sigma_{+}\left(s_{1}\right)=(\mathscr{L}+\lambda)(E) .
$$

Then a standard argument shows that the identity $\widehat{\mathscr{L}}\left(\sigma^{-1}(E)\right)=(\mathscr{L}+\lambda)(E)$ holds for every open set $E$, and hence also for every Borel set $E$. Thus (i) is proved.

In order to prove statement (ii), we first notice that by the choice of $F$ we have that $\mathbf{1}_{F} \cdot(\mathscr{L}+\lambda)=\lambda$ and $\mathbf{1}_{I \backslash F} \cdot(\mathscr{L}+\lambda)=\mathscr{L}$, and therefore statement (i) yields

$$
\sigma_{\#}\left(\mathbf{1}_{\widehat{F}} \cdot \widehat{\mathscr{L}}\right)=\mathscr{L} \quad \text { and } \quad \sigma_{\#}\left(\mathbf{1}_{\widehat{I} \backslash \widehat{F}} \cdot \widehat{\mathscr{L}}\right)=\lambda
$$

Since $\lambda$ is singular with respect to $\mathscr{L}$, it is well known that the density of $\lambda$ with respect to $\mathscr{L}$ is equal to 0 at $\mathscr{L}$-a.e. point, and equal to $+\infty$ at $\lambda$-a.e. point. This means that the derivative of the monotone functions $\sigma_{-}$and $\sigma_{+}$are 
equal to 1 at $\mathscr{L}$-a.e. point, and equal to $+\infty$ at $\lambda$-a.e. point. Therefore, taking into account $(2.10)$, we obtain that the derivative of $\sigma$ is equal to 1 at $\widehat{\mathscr{L}}$-a.e. point in $\widehat{I} \backslash \widehat{F}$, and equal to 0 at $\widehat{\mathscr{L}}$-a.e. point in $\widehat{F}$.

2.18. Remark. The definitions of $\sigma$ and $\hat{\sigma}$ and Lemma 2.17 can be extended with few obvious modification to the case where the intervals $[0, L]$ and $[0, \widehat{L}]$ are replaced by the quotients $[0, L]^{*}$ and $[0, \widehat{L}]^{*}$, see $\$ 2.10$.

We conclude this section with a short list of notation that will be frequently used in the following. Given a Lipschitz map $f: \mathbb{R}^{d} \rightarrow \mathbb{R}^{d-k}$ we have defined:

$J$ Jacobian of $f(2.5)$

$S$ critical set of $f(\$ 2.5)$;

$E_{y}:=f^{-1}(y)$ with $y \in \mathbb{R}^{d-k}$, level set of $f(\$ 2.5)$;

$\mathscr{C}_{y}$ collection of connected components $C$ of $E_{y}$ with $\mathscr{H}^{k}(C)>0$ (\$2.5);

$E^{*} \quad$ union of all $C$ with $C \in \mathscr{C}_{y}$ and $y \in \mathbb{R}^{d-k}(\$ 2.5)$;

$\nu:=\mathscr{L}^{d-k}+\nu^{s}$, measure such that $f_{\#} \mathscr{L}^{d} \ll \nu(2.7) ;$

$\mu_{y}$ disintegration of $\mathscr{L}^{d}$ with respect to $f$ and $\nu$ corresponding to the level set $E_{y}(2.7)$;

$\mu_{y}^{s}$ disintegration of the restriction of $\mathscr{L}^{d}$ to $S$ with respect to $f$ and $\nu$ (Lemma 2.8).

In the specific case of a Lipschitz function $f: \mathbb{R}^{2} \rightarrow \mathbb{R}$ we have:

$J=|\nabla f|(2.9) ;$

$S$ set of points where $f$ is not differentiable or $\nabla f=0(\$ 2.9)$;

$\mathscr{C}_{y}$ collection of nontrivial connected components of $E_{y}$ (\$2.9);

$\gamma:[0, L]^{*} \rightarrow C$, parametrization of $C \in \mathscr{C}_{y}$ given in Lemma 2.11.

$\mathscr{L}$ Lebegsue measure on $[0, L]^{*}(\$ 2.10)$;

$\lambda$ measure on $[0, L]^{*}$ defined in Lemma 2.11

\section{Dimension REDUCTION}

We now begin to consider the question of the uniqueness for the Cauchy problem for the continuity equation (1.1). In this section, we still work in general space dimension. The existence of a Lipschitz function associated to the vector field as in $\$ 3.1$ gives the possibility to relate the uniqueness for the Cauchy problem in $\mathbb{R}^{d}$ with the uniqueness for suitably defined Cauchy problems on the level sets of such Lipschitz function (Theorem 3.10).

3.1. Assumptions on the vector field. In this section $b:[0, T) \times \mathbb{R}^{d} \rightarrow \mathbb{R}^{d}$ is a bounded vector field and we assume that there exists a Lipschitz function $f: \mathbb{R}^{d} \rightarrow \mathbb{R}^{d-k}$ with compact support such that

$$
\nabla f(x) \cdot b(t, x)=0 \quad \text { for a.e. }(t, x) \in[0, T) \times \mathbb{R}^{d} .
$$

Further assumptions on $b$ and $f$ will be introduced when needed. 
3.2. The uniqueness problem. By linearity, the uniqueness for the Cauchy problem for (1.1) is equivalent to the fact that every solution in the sense of distributions with initial value $u_{0}=0$ is trivial, that is, a.e. null in $[0, T) \times \mathbb{R}^{d}$. Therefore, in this and in the following sections, we only consider solutions of the Cauchy problem

$$
\left\{\begin{array}{l}
\partial_{t} u+\operatorname{div}(b u)=0 \\
u(0, \cdot)=0
\end{array}\right.
$$

We recall that a bounded function $u:[0, T) \times \mathbb{R}^{d} \rightarrow \mathbb{R}$ solves $(3.2)$ in the sense of distributions if

$$
\int_{0}^{T} \int_{\mathbb{R}^{d}}\left(\partial_{t} \varphi+b \cdot \nabla \varphi\right) u d x d t=0 \quad \forall \varphi \in C_{c}^{\infty}\left([0, T) \times \mathbb{R}^{d}\right) .
$$

3.3. Remark. In the following we will (often tacitly) use that the test functions in (3.3) can be equivalently taken in any of the following classes:

(i) $\varphi:[0, T) \times \mathbb{R}^{d} \rightarrow \mathbb{R}$ Lipschitz with compact support;

(ii) $\varphi$ of the form $\varphi_{1}(t) \varphi_{2}(x)$, with $\varphi_{1} \in C_{c}^{\infty}([0, T))$ and $\varphi_{2} \in C_{c}^{\infty}\left(\mathbb{R}^{d}\right)$;

(iii) $\varphi$ of the form $\varphi_{1}(t) \varphi_{2}(x)$, with $\varphi_{1}:[0, T) \rightarrow \mathbb{R}$ Lipschitz with compact support and $\varphi_{2}: \mathbb{R}^{d} \rightarrow \mathbb{R}$ Lipschitz with compact support.

3.4. Remark. Here and in the rest of the paper $u$ and $b$ are functions defined at every point of their domain, and not equivalence classes 15 The reason is that it is more convenient to use the disintegration formula (2.3) with functions defined everywhere instead than almost everywhere.

3.5. Measure solutions. For technical reasons it is convenient to introduce the following notion of generalized solution of $(3.2) \sqrt{16}$ We say that a signed locally finite measure $\eta$ on $[0, T) \times \mathbb{R}^{d}$ is a measure solution of $(3.2)$ if

$$
\int_{[0, T) \times \mathbb{R}^{d}}\left(\partial_{t} \varphi+b \cdot \nabla \varphi\right) d \eta=0 \quad \forall \varphi \in C_{c}^{\infty}\left([0, T) \times \mathbb{R}^{d}\right) .
$$

3.6. Solutions on level sets. Take $y \in \mathbb{R}^{d-k}$ and let $C$ be a subset of the level set $E_{y}$. We say that a bounded function $u:[0, T) \times C \rightarrow \mathbb{R}$ is a solution of (3.2) on $C$ if the measure $\eta=u \mathbf{1}_{C} \cdot \mathscr{L}^{1} \otimes \mu_{y}$ is a measure solution in the sense of $\$ 3.5$, that is if

$$
\int_{0}^{T} \int_{C}\left(\partial_{t} \varphi+b \cdot \nabla \varphi\right) u d \mu_{y} d t=0 \quad \forall \varphi \in C_{c}^{\infty}\left([0, T) \times \mathbb{R}^{d}\right) .
$$

It is important to keep in mind that this notion of solution does not involve only the function $u$ and the set $C$, but also the disintegration measure $\mu_{y}$.

The following lemma shows that any bounded solution of $(3.2)$ is also a solution on a generic level set.

\footnotetext{
15 Accordingly, we avoid the notations $u \in L^{\infty}$ and $b \in L^{\infty}$.

${ }^{16}$ This notion is similar but not equivalent to that of measure solution considered for instance in $[6$.
} 
3.7. Lemma. Let $u:[0, T) \times \mathbb{R}^{d} \rightarrow \mathbb{R}$ be a bounded solution of $(3.2)$. Then $u$ solves $(3.2)$ on the level set $E_{y}$ for $\nu$-a.e. $y \in \mathbb{R}^{d-k}$.

Proof. Fix a function $\rho \in C_{c}^{\infty}\left(\mathbb{R}^{d-k}\right)$. We consider in (3.3) Lipschitz test functions of the form $\varphi(t, x) \rho(f(x))$, where $f: \mathbb{R}^{d} \rightarrow \mathbb{R}^{d-k}$ is the Lipschitz function introduced in $\$ 3.1$ (recall Remark 3.3(i)). After immediate computations we obtain

$$
\begin{aligned}
0 & =\int_{0}^{T} \int_{\mathbb{R}^{d}}\left[\rho(f) \partial_{t} \varphi+\rho(f) b \cdot \nabla \varphi+\varphi\langle\nabla f \cdot b ; \nabla \rho(f)\rangle\right] u d x d t \\
& =\int_{0}^{T} \int_{\mathbb{R}^{d}} \rho(f)\left(\partial_{t} \varphi+b \cdot \nabla \varphi\right) u d x d t=0,
\end{aligned}
$$

where the last equality follows from (3.1).

Using (2.3), the above integral rewrites as

$$
\begin{aligned}
0 & =\int_{\mathbb{R}^{d-k}} \int_{0}^{T} \int_{E_{y}} \rho(f)\left(\partial_{t} \varphi+b \cdot \nabla \varphi\right) u d \mu_{y}(x) d t d \nu(y) \\
& =\int_{\mathbb{R}^{d-k}} \rho(y)\left[\int_{0}^{T} \int_{E_{y}}\left(\partial_{t} \varphi+b \cdot \nabla \varphi\right) u d \mu_{y}(x) d t\right] d \nu(y)=0 .
\end{aligned}
$$

The arbitrariness of $\rho$ gives the existence of a $\nu$-negligible set $N_{\varphi} \subset \mathbb{R}^{d-k}$ with the property that

$$
\int_{0}^{T} \int_{E_{y}}\left(\partial_{t} \varphi+b \cdot \nabla \varphi\right) u d \mu_{y}(x) d t=0 \quad \text { for all } y \notin N_{\varphi} .
$$

Finally, we consider a countable dense set $\mathscr{D} \subset C_{c}^{\infty}\left([0, T) \times \mathbb{R}^{d}\right)$ and set $N=\cup_{\varphi \in \mathscr{D}} N_{\varphi}$. Since $\mathscr{D}$ is countable the set $N$ is $\nu$-negligible. Moreover, for all $\varphi \in D$ there holds

$$
\int_{0}^{T} \int_{E_{y}}\left(\partial_{t} \varphi+b \cdot \nabla \varphi\right) u d \mu_{y}(x) d t=0 \quad \text { for all } y \notin N ;
$$

this fact and the density of $\mathscr{D}$ in $C_{c}^{\infty}\left([0, T) \times \mathbb{R}^{d}\right)$ imply the result.

3.8. Lemma. Take $y \in \mathbb{R}^{d-k}$ with $y \neq 0$ and let $u:[0, T) \times E_{y} \rightarrow \mathbb{R}$ be a bounded solution of (3.2) on $E_{y}$. Then $u$ solves (3.2) on every connected component $C$ of $E_{y}$.

Proof. The fact that $u$ is a solution of $(3.2)$ on $E_{y}$ means that (recall $\left.\$ 3.6\right)$

$$
\int_{0}^{T} \int_{E_{y}}\left(\partial_{t} \varphi+b \cdot \nabla \varphi\right) u d \mu_{y} d t=0 \quad \forall \varphi \in C_{c}^{\infty}\left([0, T) \times \mathbb{R}^{d}\right) .
$$

Given a connected component $C$ of $E_{y}$, it is possible to find a decreasing sequence of bounded open sets $U_{n}$ contained in $\mathbb{R}^{d}$ such that the boundaries $\partial U_{n}$ do not intersect $E_{y}$, and the intersection of the closures $\bar{U}_{n}$ is $C$ (a proof of this fact is briefly sketched in [2, $\S 2.8]$ ). 
Let $\varepsilon_{n}$ be the distance between the sets $\partial U_{n}$ and $E_{y}$ (it is positive because these sets are closed and disjoint). Given a smooth convolution kernel $\rho$ supported in the unit ball of $\mathbb{R}^{d}$ we define

$$
\chi_{n}:=\mathbf{1}_{U_{n}} * \rho_{\varepsilon_{n} / 2} .
$$

Considering in (3.6) test functions of the form $\varphi(t, x) \chi_{n}(x)$, after standard computations we obtain

$$
\int_{0}^{T} \int_{E_{y}} \chi_{n}\left(\partial_{t} \varphi+b \cdot \nabla \varphi\right) u d \mu_{y} d t+\int_{0}^{T} \int_{E_{y}} \varphi b \cdot \nabla \chi_{n} u d \mu_{y} d t=0 .
$$

Now we pass to the limit as $n \rightarrow+\infty$ in this equality. The definition of $\chi_{n}$ and the properties of the sets $U_{n}$ give that $\nabla \chi_{n}=0$ on $E_{y}$ for every $n$, so that the second integral in (3.7) vanishes; concerning the first integral, we have that $\chi_{n}$ converge to $\mathbf{1}_{C}$ everywhere in $E_{y}$, and therefore, using the dominated convergence theorem,

$$
\int_{0}^{T} \int_{C}\left(\partial_{t} \varphi+b \cdot \nabla \varphi\right) u d \mu_{y} d t=0
$$

for all $\varphi \in C_{c}^{\infty}\left([0, T) \times \mathbb{R}^{d}\right)$, that is, $u$ solves $(3.2)$ on $C$.

3.9. Lemma. Given a bounded function $u:[0, T) \times \mathbb{R}^{d} \rightarrow \mathbb{R}$, the following statements are equivalent:

(a) 4 solves (3.2);

(b) there exists an $\mathscr{L}^{d-k}$-negligible set $N \subset \mathbb{R}^{d-k}$ such that $N$ supports $\nu^{s}$, and setting $F:=f^{-1}(N) \cup\left(\mathbb{R}^{d} \backslash E^{*}\right)$ with $E^{*}$ given in $\$ 2.5$ there holds

(b1) u solves (3.2) on $C$ for every $C \in \mathscr{C}_{y}$ and every $y \notin N$;

(b2) $\mathbf{1}_{F}$ u solves $(3.2)$.

Proof. Step 1: (a) implies (b). Since $\nu=\mathscr{L}^{d-k}+\nu^{s}$, Lemma 3.7 implies that $u$ solves 3.2 on $E_{y}$ for $\mathscr{L}^{d-k}$-a.e. $y$. Therefore, recalling Lemma 2.6(ii), we can choose an $\mathscr{L}^{d-k}$-negligible set $N$ so that

(i) $0 \in N$;

(ii) $N$ supports $\nu^{s}$;

(iii) $u$ solves $(3.2)$ on $E_{y}$ for every $y \notin N$;

(iv) $\mathscr{H}^{k}\left(E_{y}\right)<+\infty$ for every $y \notin N$.

Then, (b1) follows from Lemma 3.8. It remains to prove (b2).

Let $y \notin N$ be fixed for the time being. Because of (iv), $\mathscr{C}_{y}$ is countable, and therefore, by summing (3.5) over all $C \in \mathscr{C}_{y}$ and recalling that their union is $E_{y}^{*}$, we obtain

$$
\int_{0}^{T}\left[\int_{E_{y}^{*}}\left(\partial_{t} \varphi+b \cdot \nabla \varphi\right) u d \mu_{y}\right] d t=0 \quad \forall \varphi \in C_{c}^{\infty}\left([0, T) \times \mathbb{R}^{d}\right),
$$


that is, $u$ solves $(3.2)$ on $E_{y}^{*}$. By integrating (3.8) over all $y \notin N$ with respect to $\nu$ we obtain

$$
\begin{aligned}
0 & =\int_{0}^{T}\left[\int_{\mathbb{R}^{d-k} \backslash N} \int_{E_{y}^{*}}\left(\partial_{t} \varphi+b \cdot \nabla \varphi\right) u d \mu_{y} d \nu(y)\right] d t \\
& =\int_{0}^{T}\left[\int_{\mathbb{R}^{d} \backslash F}\left(\partial_{t} \varphi+b \cdot \nabla \varphi\right) u d x\right] d t .
\end{aligned}
$$

The second equality in the previous formula follows by the fact that $\left\{\mu_{y}\right\}$ is the disintegration of $\mathscr{L}^{d}$ with respect to $f$ and $\nu$, and by the fact that $E_{y}^{*}$ is equal to $\left(\mathbb{R}^{d} \backslash F\right) \cap E_{y}$ for all $y \notin N$.

Thus we have proved that $u \mathbf{1}_{\mathbb{R}^{d} \backslash F}$ solves $(3.2)$, and by linearity we obtain that also $u \mathbf{1}_{F}=u-u \mathbf{1}_{\mathbb{R}^{d} \backslash F}$ solves (3.2), that is, we obtain (b2).

Step 2: (b) implies (a). Let $N^{\prime}$ be the set of all $y \notin N$ such that $\mathscr{H}^{k}\left(E_{y}\right)=$ $+\infty$. We know by Lemma 2.6(ii) that $\mathscr{L}^{d-k}\left(N^{\prime}\right)=0$, and therefore $\nu\left(N^{\prime}\right)=0$ (because $\nu=\mathscr{L}^{d-k}+\nu^{s}$ and $\nu^{s}$ is supported on $N$ ).

Fix $y \notin N \cup N^{\prime}$. By (b1), $u$ solves (3.2) on every $C \in \mathscr{C}_{y}$, and since $\mathscr{H}^{k}\left(E_{y}\right)$ is finite there are only countably many such $C$. Hence, proceeding as in Step 1, we obtain that $u$ solves $(3.2)$ on $E_{y}^{*}$ and $u \mathbf{1}_{\mathbb{R}^{d} \backslash F}$ solves (3.2). Finally (b2) and the linearity of $(3.2)$ imply that $u=u \mathbf{1}_{F}+u \mathbf{1}_{\mathbb{R}^{d} \backslash F}$ solves $(3.2)$.

We can now state the main result of this section, namely that, under the additional assumption $(3.9)$, uniqueness for the Cauchy problem 3.2 in $\mathbb{R}^{d}$ is equivalent to uniqueness on the connected components $C$ in $\mathscr{C}_{y}$ for a.e. $y$.

3.10. Theorem. Take $b$ and $f$ as in 3.1 and assume that

$$
b(t, x)=0 \quad \text { for a.e. }(t, x) \in[0, T) \times S .
$$

Then the following statements are equivalent:

(a) if $u:[0, T) \times \mathbb{R}^{d} \rightarrow \mathbb{R}$ is a bounded solution of $(3.2)$ then $u=0$ for a.e. $(t, x) \in[0, T) \times \mathbb{R}^{d}$;

(b) for $\mathscr{L}^{d-k}$-a.e. $y \in \mathbb{R}^{d-k}$ and every $C \in \mathscr{C}_{y}$ the following implication holds: if $u:[0, T) \times C \rightarrow \mathbb{R}$ is a bounded solution of (3.2) on $C$, then $u=0$ for $\mathscr{L}^{1}$-a.e. $t \in[0, T)$ and $\mu_{y}$-a.e. $x \in C$.

Proof. Step 1: (b) implies (a). Let $u:[0, T) \times \mathbb{R}^{d} \rightarrow \mathbb{R}$ be a bounded solution of (3.2). We want to show that $u=0$ a.e.

From Lemma 3.9 we deduce the existence of an $\mathscr{L}^{d-k}$-negligible set $N$ such that $\nu^{s}$ is supported in $N$ and $u$ solves (3.2) on $C$ for every $C \in \mathscr{C}_{y}$ and every $y \notin N$. By assumption (b) we can find an $\mathscr{L}^{d-k}$-negligible set $N^{\prime}$ such that $u=0$ for $\mathscr{L}^{1}$-a.e. $t \in[0, T)$ and $\mu_{y}$-a.e. $x \in C$, for every $C \in \mathscr{C}_{y}$ and every $y \notin N \cup N^{\prime}$. By Lemma 2.6(ii) we can also find an $\mathscr{L}^{d-k}$-negligible set $N^{\prime \prime}$ such that $y \notin N^{\prime \prime}$ we have $\mathscr{H}^{k}\left(E_{y}\right)<+\infty$ and therefore $\mathscr{C}_{y}$ is countable.

The previous considerations imply that $u=0$ for $\mathscr{L}^{1}$-a.e. $t \in[0, T)$ and $\mu_{y}$-a.e. $x \in E_{y}^{*}$ for every $y \notin N \cup N^{\prime} \cup N^{\prime \prime}$. Recalling that $N$ support $\nu^{s}$ we 
obtain that

$$
u=0 \quad \text { a.e. in }[0, T) \times\left(E^{*} \backslash f^{-1}\left(N \cup N^{\prime} \cup N^{\prime \prime}\right)\right) .
$$

By Lemma 3.9 we know that $u \mathbf{1}_{F}$ solves $(3.2)$, where $F:=f^{-1}(N) \cup\left(\mathbb{R}^{d} \backslash E^{*}\right)$. Lemma 2.8(i) together with the fact that $N$ is an $\mathscr{L}^{d-k}$-negligible set supporting $\nu^{s}$ implies that $f^{-1}(N) \subset S$ up to negligible sets. Using Lemma 2.6(v) we deduce that $F \subset S$ up to negligible sets, thus assumption $(3.9)$ implies that $b=0$ for a.e. $(t, x) \in[0, T) \times F$. Therefore, the fact that $u \mathbf{1}_{F}$ solves 3.2 rewrites as

$$
\int_{0}^{T} \int_{F} \partial_{t} \varphi u d x d t=0 \quad \forall \varphi \in C_{c}^{\infty}\left([0, T) \times \mathbb{R}^{d}\right) .
$$

Now, since every $\phi \in C_{c}^{\infty}\left((0, T) \times \mathbb{R}^{d}\right)$ can be written as $\phi=\partial_{t} \varphi$ for some $\varphi \in C_{c}^{\infty}\left([0, T) \times \mathbb{R}^{d}\right)$, we see that (3.11) implies that

$$
u=0 \text { a.e. in }[0, T) \times F .
$$

Taking into account $(3.10)$ and $(3.12)$, to complete the proof it suffices to show that $f^{-1}\left(\left(N^{\prime} \cup N^{\prime \prime}\right) \backslash N\right)$ is negligible. This follows from the fact that $\nu\left(\left(N^{\prime} \cup N^{\prime \prime}\right) \backslash N\right)=0$ (recall $\$ 2.4$ (iii)), which in turn follows from the fact that $N^{\prime}$ and $N^{\prime \prime}$ are $\mathscr{L}^{d-k}$-negligible and $\nu^{s}$ is supported on $N$.

Step 2: (a) implies (b). Let $H$ be the set of all $y \in \mathbb{R}^{d-k}$ such that there exist $C_{y} \in \mathscr{C}_{y}$ and $\tilde{u}_{y}:[0, T) \times C_{y} \rightarrow \mathbb{R}$ a nontrivial bounded solution of (3.2) on $C_{y}$.

We have to show that $\mathscr{L}^{d-k}(H)=0$.

Assume by contradiction that $\mathscr{L}^{d-k}(H)>0$. Then we can find a constant $m<+\infty$ and a subset $H^{\prime} \subset H$ with $\mathscr{L}^{d-k}\left(H^{\prime}\right)>0$ for which the functions $\tilde{u}_{y}$ above satisfy $\left|\tilde{u}_{y}\right| \leq m$ on $[0, T) \times C_{y}$ for every $y \in H^{\prime}$.

At this point we would like to claim that the function $u$ defined by

$$
u(t, x):= \begin{cases}\tilde{u}_{y}(t, x) & \text { if } x \in C_{y} \text { for some } y \in H^{\prime} \\ 0 & \text { otherwise }\end{cases}
$$

is a nontrivial bounded solution of (3.2), in contradiction with assumption (a) of the present theorem.

Indeed, if the function $u$ were Borel, we could use the implication (b) $\Rightarrow$ (a) in Lemma 3.9 to prove such claim. Unfortunately this is not the case, and therefore we must proceed in a (slightly) different way.

We take $m$ as above and consider the set $\mathscr{C}^{*}$ of all couples $(y, \eta)$ with $y \in \mathbb{R}^{d-k}$ and $\eta$ a nontrivial signed measure on $[0, T) \times \mathbb{R}^{d}$ of the form

$$
\eta=u \cdot \mathscr{L}^{1} \otimes \mu_{y}
$$

where $u$ solves $(3.2)$ on $E_{y}$ and satisfies $|u| \leq m$ everywhere.

The projection $G$ of $\mathscr{C}^{*}$ on $\mathbb{R}^{d-k}$ contains $H^{\prime}$ because for every $y \in H^{\prime}$ the couple $\left(y, \tilde{\eta}_{y}\right)$ with $\tilde{\eta}_{y}:=\tilde{u}_{y} \mathbf{1}_{C_{y}} \cdot \mathscr{L}^{1} \otimes \mu_{y}$ belongs to $\mathscr{C}^{*}$. Hence $\mathscr{L}^{d-k}(G)>0$, 
and by Corollary 7.2 we can find a Borel set $G^{\prime} \subset G$ with

$$
\nu\left(G^{\prime}\right) \geq \mathscr{L}^{d-k}\left(G^{\prime}\right)=\mathscr{L}^{d-k}(G)>0
$$

and a Borel family of measures $\left\{\eta_{y}: y \in G^{\prime}\right\}$ so that $\left(y, \eta_{y}\right) \in \mathscr{C}^{*}$ for every $y \in G^{\prime}$.

We now denote by $u_{y}$ the bounded solution of 3.2 on $E_{y}$ associated to $\eta_{y}$ by (3.13), and we set

$$
\eta:=\int_{G^{\prime}} \eta_{y} d \nu(y)=\int_{G^{\prime}} u_{y} \cdot \mathscr{L}^{1} \otimes \mu_{y} d \nu(y) .
$$

It is clear that $\eta$ is a nontrivial measure solution of $(3.2)$, and we deduce from $\$ 2.4$ (iii), (iv) that $\eta$ is absolutely continuous with respect to $\mathscr{L}^{1} \otimes \mathscr{L}^{d}$,

Thus we can find a function $u:[0, T) \times \mathbb{R}^{d} \rightarrow \mathbb{R}$ such that $\eta=u \cdot \mathscr{L}^{1} \otimes \mathscr{L}^{d}$. Moreover, passing through the disintegration of $\eta$ with respect to $f$, we recover that for $\mathscr{L}^{d-k}$-a.e. $y \in G^{\prime}$ there holds $u=u_{y}$ a.e. with respect to $\mathscr{L}^{1} \otimes \mu_{y}$, and $u=0$ a.e. in $[0, T) \times A$, where $A$ is the complement of the union of $E_{y}$ over all $y \in G^{\prime}$.

Since $\left|u_{y}\right| \leq m$ everywhere for every $y \in G^{\prime}$ we conclude that $u$ is a nontrivial solution of $(3.2)$ which satisfies $|u| \leq m$ a.e., and this contradicts (a).

3.11. Remark. (i) The converse of Lemma 3.7 also holds: if a bounded function $u:[0, T) \times \mathbb{R}^{d} \rightarrow \mathbb{R}$ solves $(3.2)$ on the level set $E_{y}$ for $\nu$-a.e. $y \in \mathbb{R}^{d-k}$, then $u$ is a bounded solution of (3.2). This is an immediate consequence of (2.3).

(ii) The converse of Lemma 3.8 does not hold. It is not true in general that a bounded function $u:[0, T) \times E_{y} \rightarrow \mathbb{R}$ which solves $(3.2)$ on every connected component of the level set $E_{y}$ is also a solution of $(3.2)$ on the level set $E_{y}$. This is related to the fact that the connected components of a level set which are $\mathscr{H}^{k}$-negligible can in principle be more than countable.

(iii) Assumption (3.9) in Theorem 3.10 is used in the proof to show that every solution of $(3.2)$ is a.e. null on the set $F$ defined in Lemma 3.9. Presumably we could dispense with assumption (3.9); however, a proof of the corresponding variant of Theorem 3.10 would require a more refined disintegration formula, to take into account all the connected components of the level sets, including those which are $\mathscr{H}^{k}$-negligible (see the previous remark). We decided not to pursue this refinement because we did not see any specific use for it (in the two dimensional context considered in Sections 4 and 5 assumption (3.9) is always verified).

\section{Uniqueness in the aUtonomous, DiVERGenCE-FREe CASE}

4.1. Assumptions on the vector field. Through this section we assume that $d=2$ and $b: \mathbb{R}^{2} \rightarrow \mathbb{R}^{2}$ is a bounded, autonomous, divergence-free vector field with compact support. 
It follows that there exists a Lipschitz function $f: \mathbb{R}^{2} \rightarrow \mathbb{R}$ with compact support such that

$$
b=\nabla^{\perp} f \quad \text { a.e. in } \mathbb{R}^{2} \cdot 17
$$

Such $f$ is unique and is called the potential associated to $b$. Note that $b$ and $f$ satisfy the assumptions of Theorem 3.10, namely (3.1) and (3.9).

Theorem 3.10 shows that uniqueness for the Cauchy problem 3.2 is equivalent to uniqueness for the same problem on every nontrivial connected component $C$ of a generic level set $E_{y}$ of $f$. Using the parametrization of $C$ given in Lemma 2.11, we show that solving (3.2) on $C$ is equivalent to solving the Cauchy problem with zero initial datum for the equation $\partial_{t}(u(1+\lambda))+\partial_{s} u=0$ on the parametrization domain $[0, L]^{*}$ (Lemma 4.4), and for such problem there is uniqueness if and only if $\lambda=0$ (Lemma 4.5). In Theorem 4.7 we put together these results and prove that if $b$ is taken as above then uniqueness for (3.2) is equivalent to the weak Sard property for the potential $f$.

4.2. Assumptions on the set $C$. In the following $C$ is a nontrivial connected component of a level set $E_{y}$ of $f$. Taking Lemma 2.11 into account, we assume in addition that

(i) $C$ is a simple curve with finite length;

(ii) $\gamma:[0, L]^{*} \rightarrow C$ is a one-to-one Lipschitz parametrization of $C$ such that $\dot{\gamma} \neq 0$ a.e. with respect to $\mathscr{L}$ (the Lebesgue measure on $[0, L]^{*}$ );

(iii) the restriction of $\mu_{y}^{s}$ to $C$ agrees with the push-forward according to $\gamma$ of some measure $\lambda$ on $[0, L]^{*}$ which is singular with respect to $\mathscr{L}$.

The next lemma is a particular case of [2, Corollary 7.3], and states that the test functions of the form $\varphi:=\phi \circ \gamma$ with $\phi \in C_{c}^{\infty}\left(\mathbb{R}^{2}\right)$ are dense (in a suitable sense) in the class of Lipschitz functions on $[0, L]^{*}$.

4.3. Lemma (see [2], Corollary 7.3). Let a be a function in $L^{1}([0, L])$ and $\mu$ a signed measure on $[0, L]$ such that the functional

$$
\Lambda(\varphi):=\int_{0}^{L} \dot{\varphi} a d \mathscr{L}+\int_{0}^{L} \varphi d \mu
$$

satisfies $\Lambda(\varphi)=0$ for every $\varphi$ of the form $\varphi:=\phi \circ \gamma$ with $\phi \in C_{c}^{\infty}\left(\mathbb{R}^{2}\right)$.

Then $\Lambda(\varphi)=0$ for every Lipschitz function $\varphi$ on $[0, L]^{*}$.

4.4. Lemma. Given a bounded function $u:[0, T) \times C \rightarrow \mathbb{R}$, the following statements are equivalent:

(a) $u$ solves (3.2) on $C$;

(b) the function $\tilde{u}(t, s):=u(t, \gamma(s))$ solves

$$
\left\{\begin{array}{l}
\partial_{t}(\tilde{u}(1+\lambda))+\partial_{s} \tilde{u}=0 \\
\tilde{u}(0, \cdot)=0
\end{array}\right.
$$

\footnotetext{
17 Since $\operatorname{div} b=0$, by rotating $b$ by $90^{\circ}$ clockwise we obtain a curl-free vector field, which can be rewritten as the gradient of a Lipschitz scalar function.
} 
in the sense of distributions on $[0, T) \times[0, L]^{*}$, that is

$$
\int_{0}^{T}\left[\int_{0}^{L}\left(\partial_{t} \varphi+\partial_{s} \varphi\right) \tilde{u} d s+\int_{0}^{L} \partial_{t} \varphi \tilde{u} d \lambda\right] d t=0 .
$$

for every $\varphi \in C_{c}^{\infty}\left([0, T) \times[0, L]^{*}\right)[18$

Proof. Step 1: (a) implies (b). Since $\mu_{y}=\gamma_{\#}(\mathscr{L}+\lambda)$ and $b=0$ for $\gamma_{\#} \lambda$ a.e. point of $C$, the fact that $u$ solves $(3.2)$ on $C$ amounts to

$$
\int_{0}^{T} \int_{C}\left(\dot{\varphi}_{1} \varphi_{2}+\varphi_{1} b \cdot \nabla \varphi_{2}\right) u d\left(\gamma_{\#} \mathscr{L}\right) d t+\int_{0}^{T} \int_{C} \dot{\varphi}_{1} \varphi_{2} u d\left(\gamma_{\#} \lambda\right) d t=0
$$

for all $\varphi_{1} \in C_{c}^{\infty}([0, T))$ and all $\varphi_{2} \in C_{c}^{\infty}\left(\mathbb{R}^{2}\right)$.

We set $\tilde{\varphi}_{2}(s)=\varphi_{2}(\gamma(s))$ and compute

$$
\begin{aligned}
\dot{\tilde{\varphi}}_{2}(s)=\nabla \varphi_{2}(\gamma(s)) \cdot \dot{\gamma}(s)=\nabla \varphi_{2}(\gamma(s)) \cdot \nabla^{\perp} f(\gamma(s)) & \\
& \text { for } \mathscr{L} \text {-a.e. } s \in[0, L]^{*} .
\end{aligned}
$$

Using (4.5) and the change of variable $x=\gamma(s)$, identity (4.4) can be rewritten as

$$
\int_{0}^{L}\left[\int_{0}^{T} \varphi_{1} \tilde{u} d t\right] \dot{\tilde{\varphi}}_{2} d s+\int_{0}^{L}\left[\int_{0}^{T} \dot{\varphi}_{1} \tilde{u} d t\right] \tilde{\varphi}_{2}(d s+d \lambda)=0
$$

for all $\varphi_{1} \in C_{c}^{\infty}([0, T))$ and all $\tilde{\varphi}_{2}$ of the form $\tilde{\varphi}_{2}=\varphi_{2} \circ \gamma$ with $\varphi_{2} \in C_{c}^{\infty}\left(\mathbb{R}^{d}\right)$.

Then Lemma 4.3 implies that (4.6) holds also for all Lipschitz functions $\tilde{\varphi}_{2}$ on $[0, L]^{*}$, which is enough to deduce (4.3) and obtain (b).

Step 2: (b) implies (a). To show that $u$ solves (3.2) on $C$ - that is, it satisfies (3.5) - it suffices to observe that (4.3) holds also for all test functions of the form $\varphi(t, \gamma(s))$ with $\varphi:[0, T) \times C \rightarrow \mathbb{R}$ a Lipschitz function with compact support, and then apply the change of variable $x=\gamma(s)$ to the integral at the left-hand side as in the previous step.

4.5. Lemma. Take $L>0$ and let $\lambda$ be any measure on $[0, L]^{*}$ which is singular with respect to $\mathscr{L}$. Then the following statements are equivalent:

(a) if $v:[0, T) \times[0, L]^{*} \rightarrow \mathbb{R}$ is a bounded solution of 4.2 then $v=0$ for $\mathscr{L}^{1}$-a.e. $t \in[0, T)$ and $(\mathscr{L}+\lambda)$-a.e. $s \in[0, L]^{*}$;

(b) $\lambda=0$.

Proof. Step 1: (b) implies (a). If $\lambda=0$, then (4.2) reduces to

$$
\left\{\begin{array}{l}
\partial_{t} v+\partial_{s} v=0 \\
v(0, \cdot)=0
\end{array}\right.
$$

which has only the trivial solution 19

\footnotetext{
${ }^{18} C_{c}^{\infty}\left([0, T) \times[0, L]^{*}\right)$ corresponds to the space of smooth functions on $[0, T) \times \mathbb{R}$ which are $L$-periodic in the second variable and compactly supported in the first variable.

19 This is well-known with $[0, L]^{*}$ replaced by $\mathbb{R}$; the same proof works also for $[0, L]^{*}$.
} 
Step 2: (a) implies (b). We show that for $\lambda \neq 0$ the problem 4.2 has a nontrivial bounded solution. More precisely, we construct two distinct bounded solutions of the equation

$$
\partial_{t}(v(1+\lambda))+\partial_{s} v=0
$$

with initial condition $v(0, \cdot)=\mathbf{1}_{A}$, where $A$ is an $\mathscr{L}$-negligible compact set contained in $(0, L)$ with $\lambda(A)>0.20$

It is immediate to check that $v(t, s):=\mathbf{1}_{A}(s)$ is a stationary solution of 4.7$)$ with initial condition $v(0, \cdot)=\mathbf{1}_{A}$.

Consider now the function $\sigma:[0, L]^{*} \rightarrow[0, \widehat{L}]^{*}$ and its inverse $\hat{\sigma}$ defined in 2.16 (see also Remark 2.18): since the (distributional) derivative of $\hat{\sigma}$ is $1+\lambda$, a formal computation shows that the change of variable $s=\sigma(\hat{s})$ reduces the equation

$$
\partial_{t} w+\partial_{\hat{s}} w=0
$$

to (4.7). This suggests a way to construct the second solution we are looking for: set

$$
v(t, s):= \begin{cases}w(t, \hat{\sigma}(s)) & \text { for } s \notin \mathscr{A} \\ f_{\hat{\sigma}(s)} w(t, \hat{s}) d \hat{s} & \text { for } s \in \mathscr{A},\end{cases}
$$

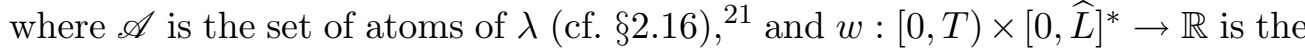
(unique) bounded solution of (4.8) with initial condition $w(0, \cdot)=\mathbf{1}_{\hat{\sigma}(A)}$, which means that $w$ satisfies

$$
\int_{0}^{T} \int_{0}^{\widehat{L}}\left(\partial_{t} \varphi+\partial_{\hat{s}} \varphi\right) w d \hat{s} d t=\int_{0}^{\widehat{L}} \varphi(0, \cdot) \mathbf{1}_{\hat{\sigma}(A)} d \hat{s}
$$

for every $\varphi \in C_{c}^{\infty}\left([0, T) \times[0, \widehat{L}]^{*}\right)$. We claim that $v$ is a solution of 4.7 with initial condition $v(0, \cdot)=\mathbf{1}_{A}$ and is not stationary, so that it differs from the stationary solution.

Step 3: $v$ solves (4.7) with initial condition $v(0, \cdot)=\mathbf{1}_{A}$. Let $F$ be an $\mathscr{L}$ negligible set which supports $\lambda$. Then the claim can be rewritten as follows: for every test function $\varphi \in C_{c}^{\infty}\left([0, T) \times[0, L]^{*}\right)$ there holds

$$
\int_{0}^{T} \int_{0}^{L}\left(\partial_{t} \varphi+\mathbf{1}_{I \backslash F} \partial_{s} \varphi\right) v d(\mathscr{L}+\lambda) d t=\int_{0}^{L} \varphi(0, \cdot) \mathbf{1}_{A} d(\mathscr{L}+\lambda) .
$$

We apply the change of variable $s=\sigma(\hat{s})$ : letting $\hat{v}(t, \hat{s}):=v(t, \sigma(\hat{s}))$ and $\hat{\varphi}(t, \hat{s}):=\varphi(t, \sigma(\hat{s}))$ and using (2.9), identity (4.11) becomes

$$
\int_{0}^{T} \int_{0}^{\widehat{L}}\left(\partial_{t} \hat{\varphi}+\partial_{\hat{s}} \hat{\varphi}\right) \hat{v} d \hat{s} d t=\int_{0}^{\widehat{L}} \hat{\varphi}(0, \cdot) \mathbf{1}_{\hat{\sigma}(A)} d \hat{s}
$$

(note that $\partial_{t} \hat{\varphi}=\partial_{t} \varphi$ and $\partial_{\hat{s}} \hat{\varphi}=\partial_{s} \varphi \mathbf{1}_{\widehat{I} \backslash \hat{\sigma}(F)}$ ).

\footnotetext{
${ }^{20}$ Such $A$ can be found because the measure $\lambda$ is supported in $(0, L)$, cf. $\$ 2.12$ (ii).

${ }^{21}$ Recall that $\hat{\sigma}$ is uni-valued for $s \notin \mathscr{A}$, and $\hat{\sigma}(s)$ is the interval $\left[\hat{\sigma}_{-}(s), \hat{\sigma}_{+}(s)\right]$ for $s \in \mathscr{A}$, cf. \$2.16
} 
If $\hat{v}$ were equal to $w$, then 4.12 would follow by writing 4.10 with the test function $\hat{\varphi}$ (recall Remark 3.3). Unfortunately, $\hat{v}$ agrees with $w$ only on the complement of $\hat{\sigma}(\mathscr{A})$. However, we can recover 4.12 from 4.10 by showing that for every $t \in[0, T)$ there holds

$$
\int_{\hat{\sigma}(\mathscr{A})}\left(\partial_{t} \hat{\varphi}+\partial_{\hat{s}} \hat{\varphi}\right) \hat{v} d \hat{s}=\int_{\hat{\sigma}(\mathscr{A})}\left(\partial_{t} \hat{\varphi}+\partial_{\hat{s}} \hat{\varphi}\right) w d \hat{s} .
$$

Indeed

$$
\begin{aligned}
\int_{\hat{\sigma}(\mathscr{A})}\left(\partial_{t} \hat{\varphi}\right. & \left.+\partial_{\hat{s}} \hat{\varphi}\right) \hat{v} d \hat{s} \\
& =\sum_{s \in \mathscr{A}} \int_{\hat{\sigma}(s)}\left(\partial_{t} \hat{\varphi}+\partial_{\hat{s}} \hat{\varphi}\right) \hat{v} d \hat{s} \\
& =\sum_{s \in \mathscr{A}} \partial_{t} \varphi(t, s) \int_{\hat{\sigma}(s)} \hat{v} d \hat{s} \\
& =\sum_{s \in \mathscr{A}} \partial_{t} \varphi(t, s) \int_{\hat{\sigma}(s)} w d \hat{s}=\int_{\hat{\sigma}(\mathscr{A})}\left(\partial_{t} \hat{\varphi}+\partial_{\hat{s}} \hat{\varphi}\right) w d \hat{s}
\end{aligned}
$$

where the second and the last equalities follow from the fact that $\partial_{\hat{s}} \hat{\varphi}(t, \hat{s})=0$ and $\partial_{t} \hat{\varphi}(t, \hat{s})=\partial_{t} \varphi(t, s)$ for all $\hat{s}$ in the interval $\hat{\sigma}(s)$, while the third equality follows from that fact that in each of these intervals the function $\hat{v}(t, \hat{s})$ coincides with the average of $w(t, \hat{s})$ over the same interval (see (4.9p).

Step 4: $v$ is not stationary. This claim may be proved in many ways. We choose to look at the maximum $a(t)$ of the support of the function $v(t, \cdot)(\mathrm{com}-$ puted with respect to the measure $\mathscr{L}+\lambda$ ), and show that this function is strictly increasing at $t=0$.

Let $\hat{a}(t)$ be the maximum of the support of the function $w(t, \cdot)$ (computed with respect to the Lebesgue measure). Since $w$ solves (4.8), a continuity equation with a regular (in fact, constant) vector field, then it is propagated along characteristics, and therefore $\hat{a}$ is strictly increasing in $t$, at least as long as it does not reach the value $L$.

Moreover $a=\sigma(\hat{a})$. Therefore, recalling the properties of $\sigma$ (see \$2.16), we obtain that $a$ is strictly increasing at $t=0$ provided that $\hat{a}(0)$ does not belong to any of the intervals $\sigma(s)$ with $s \in \mathscr{A}$, or, if it does, then it agrees with the supremum of such interval. Finally, one can check that this condition is always verified (recall that is $A$ closed).

4.6. Remark. Concerning the previous proof, it should be noted that the change of variable $s=\sigma(\hat{s})$ reduces to 4.7) every equation of the form

$$
\partial_{t} v+\partial_{\hat{s}}\left(g_{1} v+g_{2}\right)=0
$$

where the functions $g_{1}$ and $g_{2}$ satisfy the following conditions: $g_{1}$ is constant a.e. in $\hat{\sigma}(F), g_{1}=1$ and $g_{2}=0$ a.e. outside $\hat{\sigma}(F)$. More precisely, if $w$ is a solution of (4.14), then the function $v$ defined by (4.9) is a solution of (4.7). 
In particular, taking $g_{1}:=1$ and $g_{2}:=-\mathbf{1}_{A}$ we recover the stationary solution $v(t, s):=\mathbf{1}_{A}(s)$.

4.7. Theorem. Take $b$ and $f$ as in $\$ 4.1$. Then the following statements are equivalent:

(a) if $u:[0, T) \times \mathbb{R}^{2} \rightarrow \mathbb{R}$ is a bounded solution of $(3.2)$ then $u=0$ for a.e. $(t, x) \in[0, T) \times \mathbb{R}^{2}$

(b) the potential $f$ satisfies the weak Sard property (defined in \$2.13).

Proof. Since $b$ and $f$ satisfy the assumptions of Theorem 3.10, the uniqueness for the Cauchy problem (3.2) (statement (a)) is equivalent to uniqueness for the same problem on every $C \in \mathscr{C}_{y}$ for a.e. $y$.

By Lemma 2.11, for a.e. $y$, every connected component $C \in \mathscr{C}_{y}$ satisfies the assumptions in \$4.2, and therefore Lemma 4.4 and Lemma 4.5 imply that the uniqueness of (3.2) on $C$ is equivalent to $\lambda=0$, which means that the restriction of $\mu_{y}^{s}$ to $C$ vanishes.

Finally Lemma 2.14 states that $\mathbf{1}_{C} \cdot \mu_{y}^{s}=0$ for every $C \in \mathscr{C}_{y}$ and a.e. $y$ if and only if $f$ satisfies the weak Sard property.

4.8. Corollary. There exists a divergence-free autonomous vector field $b$ on the plane which belongs to $C^{0, \alpha}$ for every $\alpha<1$, and for which the Cauchy problem (3.2) has nontrivial bounded solutions.

Proof. In $[2, \S 4.8]$ we construct a function $f^{\prime}: \mathbb{R}^{2} \rightarrow \mathbb{R}$ of class $C^{1, \alpha}$ for every $\alpha<1$ which does not have the weak Sard property. We modify $f^{\prime}$ so to make it compactly supported, and set $b:=\nabla^{\perp} f^{\prime}$. Then Theorem 4.7 shows that for such $b$ the Cauchy problem (3.2) admits nontrivial bounded solutions.

\section{UNIQUENESS IN THE BOUNDED DIVERGENCE CASE}

5.1. Assumptions on the vector field. In this section we consider a bounded, time-dependent vector field $b:[0, T) \times \mathbb{R}^{2} \rightarrow \mathbb{R}^{2}$ of the form

$$
b(t, x)=a(t, x) \nabla^{\perp} f(x) \quad \text { a.e. in }[0, T) \times \mathbb{R}^{2},
$$

where $f: \mathbb{R}^{2} \rightarrow \mathbb{R}$ is a Lipschitz function with compact support and $a:[0, T) \times$ $\mathbb{R}^{2} \rightarrow \mathbb{R}$ is a bounded function.

We assume moreover that $b$ has bounded divergence in the sense of distributions, that is, there exists a bounded function $g:[0, T) \times \mathbb{R}^{2} \rightarrow \mathbb{R}$ such that

$$
\int_{0}^{T} \int_{\mathbb{R}^{2}} b \cdot \nabla \varphi+g \varphi d x d t=0 \quad \forall \varphi \in C_{c}^{\infty}\left((0, T) \times \mathbb{R}^{2}\right) .
$$

The next result extends Theorem 4.7 and provides a characterization of uniqueness within the special class of bounded vector fields considered in the previous paragraph.

5.2. Theorem. Take $b, f$ and $a$ as in $\S 5.1$. The following statements hold: 
(i) If $f$ satisfies the weak Sard property then there is uniqueness for the Cauchy problem (3.2), that is, every bounded solution $u:[0, T) \times \mathbb{R}^{2} \rightarrow \mathbb{R}$ of (3.2) satisfies $u=0$ for a.e. $(t, x) \in[0, T) \times \mathbb{R}^{2}$.

(ii) Conversely, if $f$ does not satisfy the weak Sard property and there exists $\delta>0$ such that $a \geq \delta$ a.e. on $[0, T) \times \mathbb{R}^{2}$, then (3.2) admits a nontrivial bounded solution.

5.3. Remark. (i) Observe that, for given $b$ and $f$, the value of $a$ is determined (a.e.) in $[0, T) \times\left(\mathbb{R}^{2} \backslash S\right)$ by equation $(5.1)$, but can be freely chosen on $[0, T) \times S$.

(ii) The class of vector fields considered in this section clearly includes the one considered in Section 4. The structural assumption (5.1) is however very rigid, in that it allows only for vector fields whose direction does not depend on time, and does not even include all autonomous vector fields with bounded divergence ${ }^{22}$

(iii) The role of the non-degeneracy assumption $a \geq \delta>0$ in statement (ii) of Theorem 5.2 will be discussed in detail in Remark 5.5 at the end of this section. Here we just we just summarize a few conclusions:

(a) Some non-degeneracy assumption on $a$ is needed: if $b$ (or equivalently a) vanishes on a neighbourhood of the critical set $S$, then uniqueness for (3.2) holds regardless of whether $f$ satisfies the weak Sard property or not.

(b) It is not enough to assume that $a$ is strictly positive $(a>0)$.

(c) The assumption $a \geq \delta>0$ can be clearly replaced by $a \leq-\delta<0,23$ but none of these is optimal. Yet we could not find weaker conditions that are easily expressed only in terms of $a$ and $f$.

(iv) Consider two different couples $f_{1}, a_{1}$ and $f_{2}, a_{2}$ that decompose $b$ as in (5.1), that is

$$
b=a_{1} \nabla^{\perp} f_{1}=a_{2} \nabla^{\perp} f_{2} .
$$

Under the assumption that both $a_{1}$ and $a_{2}$ are positive and bounded away from zero, then $f_{1}$ satisfies the weak Sard property if and only if $f_{2}$ does (because by Theorem 5.2 both conditions are equivalent to the uniqueness for (3.2)). More generally, if $a_{2}$ is positive and bounded away from zero then the weak Sard property for $f_{1}$ implies that for $f_{2}$.

We first prove a lemma that characterizes the derivative of $a$ along a nontrivial connected component $C$ of a generic level set $E_{y}$. The rest of the proof of Theorem 5.2 is a more or less straightforward modification of the proof of Theorem 4.7, and we will only summarize the main steps.

\footnotetext{
22 The vector field $b(x):=x$ cannot be written in the form 5.1 in any neighbourhood of 0 : if it were, all level sets of $f$ would contain the point 0 , which is clearly impossible for a non-constant Lipschitz function.

23 Just apply the result with $a$ and $f$ replaced by $-a$ and $-f$.
} 
5.4. Lemma. For a.e. $y \in \mathbb{R}$, every connected component $C \in \mathscr{C}_{y}$ satisfies the assumptions in $\$ 4.2$, and

$$
\partial_{s} \tilde{a}=\tilde{g}(1+\lambda)
$$

in the sense of the distributions on $(0, T) \times[0, L]^{*}$, where we have set $\tilde{a}(t, s):=$ $a(t, \gamma(s))$ and $\tilde{g}(t, s):=g(t, \gamma(s))$ and $\gamma, \lambda, \mathscr{L}$ are taken as in $\$ 4.2$.

Moreover there exists a function $\alpha:[0, T) \times[0, \widehat{L}]^{*} \rightarrow \mathbb{R}$ which is bounded, uniformly Lipschitz in the second variable, and satisfies $\tilde{a}(t, s)=\alpha(t, \hat{\sigma}(s))$ for a.e. $(t, s)$, where $\widehat{L}$ and $\hat{\sigma}:[0, L]^{*} \rightarrow[0, \widehat{L}]^{*}$ are given in $\widehat{2.16}$ (see also Remark 2.18).

In particular when $\lambda=0$ we have $\widehat{L}=L, \hat{\sigma}(s)=s$, and therefore $\tilde{a}=\alpha$ a.e.

Sketch of PRoOF. Step 1: proof of (5.3). Following the proofs of Lemmas 3.7 and 3.8 we obtain that for every $C \in \mathscr{C}_{y}$ and a.e. $y$ there holds

$$
\int_{0}^{T} \int_{C} b \cdot \nabla \varphi+g \varphi d \mu_{y} d t=0 \quad \forall \varphi \in C_{c}^{\infty}\left((0, T) \times \mathbb{R}^{2}\right) .
$$

Moreover $C$ satisfies the requirements in $\$ 4.2$ (Lemma 2.11) and therefore, arguing as in the proof of Lemma 4.4, equation (5.4) translates into

$$
\int_{0}^{T}\left[\int_{0}^{L} \tilde{a} \partial_{s} \varphi+g \varphi d \mathscr{L}+\int_{0}^{L} \tilde{g} \varphi d \lambda\right] d t=0 \quad \forall \varphi \in C_{c}^{\infty}\left((0, T) \times[0, L]^{*}\right),
$$

which is precisely the weak formulation of (5.3).

Step 2: construction of $\alpha$. For every $t \in[0, T)$ and $\hat{s} \in[0, \widehat{L}]$ we set

$$
\alpha(t, \hat{s}):=c(t)+\int_{0}^{\hat{s}} \tilde{g}(t, \sigma(\cdot)) d \mathscr{L},
$$

and we choose $c(t)$ so that the integrals of $\tilde{a}(t, \cdot)$ and $\alpha(t, \hat{\sigma}(\cdot))$ over $[0, L]$ are the same.

The function $\alpha$ is clearly well-defined on $[0, T) \times[0, \widehat{L}]$, bounded, and Lipschitz in the second variable. Moreover (5.3) and formula (2.9) imply that for a.e. $t$

$$
0=\int_{0}^{L} \tilde{g}(t, \cdot) d(\mathscr{L}+\lambda)=\int_{0}^{\widehat{L}} \tilde{g}(t, \sigma(\cdot)) d \mathscr{L}
$$

and therefore $\alpha(t, 0)=\alpha(t, \widehat{L})$. Hence $\alpha(t, \cdot)$ is well-defined and Lipschitz also as a function on $[0, \widehat{L}]^{*}$.

Step 3. Formula (2.9) yields

$$
\alpha(t, \hat{\sigma}(s))=c(t)+\int_{0}^{s} \tilde{g}(t, \cdot) d(\mathscr{L}+\lambda)
$$

for every $t$ and every $s$ such that $\hat{\sigma}$ is uni-valued at $s$. This identity and (5.3) imply that

$$
\partial_{s} \alpha(t, \hat{\sigma}(s))=\tilde{g}(1+\lambda)=\partial_{s} \tilde{a}
$$

in the sense of distribution on $(0, T) \times[0, L]^{*}$. In turn, (5.5) and the choice of $c(t)$ imply that $\alpha(t, \hat{\sigma}(s))=\tilde{a}(t, s)$ for a.e. $(t, s)$. 
Sketch of proof of Theorem 5.2, Step 1 . Since $b$ and $f$ satisfy the assumptions of Theorem 3.10, the uniqueness for the Cauchy problem (3.2) is equivalent to uniqueness for the same problem on every $C \in \mathscr{C}_{y}$ for a.e. $y$. Moreover, in view of Lemma 5.4, we can restrict ourselves to those $C$ which satisfy the assumptions in 4.2 and in Lemma 5.4 .

Step 2. A straightforward modification of Lemma 4.4 shows that the uniqueness for the Cauchy problem $(3.2)$ on $C$ is equivalent to the uniqueness for

$$
\left\{\begin{array}{l}
\partial_{t}(\tilde{u}(1+\lambda))+\partial_{s}(\tilde{a} \tilde{u})=0 \\
\tilde{u}(0, \cdot)=0
\end{array}\right.
$$

on $[0, T) \times[0, L]^{*}$, where $\tilde{a}$ is defined as in Lemma 5.4 .

Step 3: proof of statement (i). Since $f$ satisfies the weak Sard property, by Lemma 2.14 we can assume that the restriction of $\mu_{y}^{s}$ to $C$ vanishes, which means that $\lambda$ vanishes.

By the previous steps, it suffices to show that for $\lambda=0$ the only bounded solution of (5.6) is the trivial one. Indeed (5.6) reduces to the standard continuity equation

$$
\left\{\begin{array}{l}
\partial_{t} \tilde{u}+\partial_{s}(\tilde{a} \tilde{u})=0 \\
\tilde{u}(0, \cdot)=0
\end{array}\right.
$$

Since $\tilde{a}$ agrees a.e. with the function $\alpha$ given in Lemma 5.4 , and $\alpha$ is Lipschitz in the second variable, the fact that $\tilde{u}$ is trivial follows by a standard result.

Step 4: proof of statement (ii). In view of the first two steps, it suffices to show that if $\lambda \neq 0$ then the problem (5.6) admits a nontrivial bounded solution.

To do this, we strictly follow the second part of the proof of Lemma 4.5 and construct two distinct bounded solutions of the equation

$$
\partial_{t}(v(1+\lambda))+\partial_{s}(\tilde{a} v)=0,
$$

with the same initial condition $v(0, \cdot)=\mathbf{1}_{A}$, where $A$ is chosen as in that proof.

One solution is the stationary one, and the other one is the function $v$ given by formula (4.9) by taking as $w$ the (unique) bounded solution of

$$
\partial_{t} w+\partial_{\hat{s}}(\alpha w)=0
$$

on $[0, T) \times[0, \widehat{L}]^{*}$ with initial condition $w(0, \cdot)=\mathbf{1}_{\hat{\sigma}(A)}$, where $\alpha$ is the function constructed in Lemma 5.4 .

The fact that $v$ solves (5.8) and is different from the stationary solution can be proved as in Lemma 4.5 .

5.5. Remark. The assumption $a \geq \delta>0$ in statement (ii) of Theorem 5.2 is used only once in the proof above, and precisely to show that the solution $v$ of (5.8) built out of a certain solution $w$ of $(5.9)$ is not stationary. Assuming that $a$, and therefore $\alpha$, is positive and bounded away from zero is clearly sufficient for this purpose (even though not necessary).

In the rest of this paragraph we argue that just assuming $a$ strictly positive is not enough. 
We take a function $f$ of class $C^{1}$ on $\mathbb{R}^{2}$ without the weak Sard property (see [2. Section 4]) and construct a function $a=a(x)$ so that each $\tilde{a}$ is Lipschitz, positive, and vanishes only on the closed set $\gamma^{-1}(S)$. We claim that the Cauchy problem (3.2) with $b:=a \nabla^{\perp} f$ admits only the trivial solution, despite the fact that $f$ does not satisfy the weak Sard property and $a$ is strictly positive ${ }^{24}$

As pointed out in the proof above, this is equivalent to show that (5.6) admits only the trivial solution. Now $\tilde{a}$ vanishes on $\gamma^{-1}(S)$ by construction, and since $\lambda$ is supported on $\gamma^{-1}(S)$, we have that $\tilde{a}$ vanishes $\lambda$-a.e. Therefore the equation in (5.6) can be rewritten as $\partial_{t} v+\partial_{s}(\tilde{a} v)=0$ with $v:=\tilde{u}(1+\lambda)$, and it is known that for this equation there is uniqueness also among measure solutions because $\tilde{a}$ is Lipschitz.

\section{AdDitional REMARKS}

6.1. Extensions of Theorems 4.7 and 5.2, (i) In both statements, the assumption that $b$ has compact support is made for the sake of simplicity, and can be easily removed. In that case, one should also consider nontrivial connected components $C$ of the level sets of $f$ which are simple curves with end points at infinity.

(ii) The continuity equation (1.1) can be modified so to include a (possibly nonlinear) source term at the right-hand side, that is

$$
\partial_{t} u+\operatorname{div}(b u)=h(t, x, u),
$$

where the function $h:[0, T) \times \mathbb{R}^{2} \times \mathbb{R} \rightarrow \mathbb{R}$ is bounded. The key point is clearly that uniqueness holds for the corresponding one-dimensional equation

$$
\partial_{t} v+\partial_{s}(\tilde{a} v)=\tilde{h}(t, s, v)
$$

on $[0, T) \times[0, L]^{*}$.

(iii) Uniqueness can be shown in the class of weak solutions that are integrable in space and time (instead of bounded). The key point is that uniqueness holds for the corresponding one-dimensional equation $\partial_{t} v+\partial_{s}(\tilde{a} v)=0$ among solutions which are integrable in space and time.

6.2. Extension to higher dimension. It is possible to extend Theorem 5.2 to higher dimension, and more precisely to bounded, time-dependent vector fields $b$ on $\mathbb{R}^{d}$ with bounded divergence which satisfy the following (quite rigid) structural assumption: there exists a Lipschitz map $f: \mathbb{R}^{d} \rightarrow \mathbb{R}^{d-1}$ such that $\nabla f \cdot b=0$ a.e., and $b=0$ at a.e. point where the rank of $\nabla f$ is not maximal.

In this case, the uniqueness for the Cauchy problem $(3.2)$ should be proved under the following assumptions: (i) $f$ satisfies a suitable version of the weak Sard property, and (ii) for a.e. $y$ the level set $E_{y}$ of $f$ does not contains triods, and therefore its nontrivial connected components are simple curves.

\footnotetext{
${ }^{24}$ By construction we only imposed that $a$ is strictly positive (a.e.) outside $S$, but remember that $a$ can be freely modified in $S$ without affecting $b$. The fact that $b$ has bounded divergence follows by a suitable converse of Lemma 5.4
} 
It seems that both assumptions are not only sufficient for uniqueness, but also necessary. Note that assumption (ii) is automatically satisfied when $d=2$ (Lemma 2.11(i)), while for $d>2$ it is satisfied when $f$ is of class $C^{1,1 / 2}$ (see 2, Lemma 2.16]), but may fail when $f$ is of class $C^{1, \alpha}$ with $\alpha<1 /(d-1)$ (see [2, Section 3]).

Since also in this case the uniqueness for the Cauchy problem $(3.2)$ turns out to be equivalent to the uniqueness for the same problem on the nontrivial connected components of a generic level set of $f$, the lack of triods is (presumably) also necessary for uniqueness.

6.3. Renormalization property. The key step in the uniqueness proof by DiPerna and Lions [13] is proving the renormalization property for weak solutions.

This property is not used in this paper, but it can be easily obtained as a consequence of our dimension-reduction technique. Let us assume for simplicity that $b$ is divergence-free and autonomous: in this case the renormalization property for a weak solution $u$ of $(1.1)$ simply means that $\beta(u)$ is a weak solution of the same equation for every smooth function $\beta: \mathbb{R} \rightarrow \mathbb{R}: 25$ when the potential $f$ associated to $b$ satisfies the weak Sard property this fact follows from the renormalization property for the one-dimensional equation $\partial_{t} v+\partial_{s} v=0$.

6.4. Regular Lagrangian flow. Under the weak regularity assumptions on $b$ considered in this paper, the meaningful notion of flow for the ordinary differential equation $\dot{x}=b(t, x)$ is that of regular Lagrangian flow (see [4]): we say that a Borel map $\Phi:[0, T) \times \mathbb{R}^{2} \rightarrow \mathbb{R}^{2}$ is a regular Lagrangian flow associated to $b$ if

(i) for a.e. $x \in \mathbb{R}^{2}$ the map $t \mapsto \Phi(t, x)$ solves $\dot{x}=b(t, x)$ in the integral sense;

(ii) there exists a constant $c$ such that for every $t$ the push-forward of $\mathscr{L}^{2}$ according to $\Phi_{t}:=\Phi(t, \cdot)$ satisfies $\left(\Phi_{t}\right)_{\#} \mathscr{L}^{2} \leq c \mathscr{L}^{2}$.

Theorems 4.7 and 5.2 , together with the abstract theory of regular Lagrangian flows developed in [6], give the following result: if $b$ is taken as in 4.1 or 5.1 , and the corresponding function $f$ satisfies the weak Sard property, then there exists a unique regular Lagrangian flow for $b$.

6.5. Strong locality of the divergence operator. It is well-known that the (distributional) gradient is strongly local for Sobolev functions, in the sense that the following implication holds for every Sobolev function $u$ and every Borel set $A$ in the domain of $u$ :

$$
u=\text { const. a.e. on } A \Rightarrow \nabla u=0 \text { a.e. on } A \text {. }
$$

It follows immediately that also every first-order differential operator is strongly local on Sobolev spaces.

\footnotetext{
${ }^{25}$ If $u$ is of class $C^{1}$, this follows by a straightforward computation.
} 
However, this is no longer true on larger spaces; in particular the divergence operator is not strongly local on the space of vector fields with bounded divergence. Indeed, for vector fields $b$ on $\mathbb{R}^{2}$ of the form

$$
b=a \nabla^{\perp} f
$$

where $f$ is a Lipschitz function on $\mathbb{R}^{2}$ and $a$ a bounded function on $\mathbb{R}^{2}$, the strong locality of the divergence is strictly related to the weak Sard property of $f$. More precisely:

6.6. Proposition. Let $b, f$ and $a$ be given as above, and assume that $b$ has bounded divergence. Then the following statements hold for every $v \in \mathbb{R}^{2}$ :

(i) If the function $f(x)-v \cdot x$ has the weak Sard property, then for every Borel set $A$ in $\mathbb{R}^{2}$ there holds:

$$
b=v \text { a.e. on } A \Rightarrow \operatorname{div} b=0 \text { a.e. on } A .
$$

(ii) Conversely, if $f(x)-v \cdot x$ does not satisfy the weak Sard property then there exist $b$ as above and a Borel set A such that implication (6.2) fails.

In [2, Section 5] we construct an explicit example of bounded vector field $b$ on the plane whose divergence is bounded, nontrivial, and supported in the set where $b=0$, and then use such $b$ to construct a Lipschitz function without the weak Sard property.

Sketch OF PROOF. With no loss of generality we can assume $v=0$. We first prove statement (i). Let $g$ be the divergence of $b$, that is,

$$
\int_{\mathbb{R}^{2}} b \cdot \nabla \varphi+g \varphi d x=0 \quad \forall \varphi \in C_{c}^{\infty}\left(\mathbb{R}^{2}\right) .
$$

Starting from this identity and arguing as in the proof of Lemma 3.9 we find an $\mathscr{L}^{1}$-negligible set $N$ in $\mathbb{R}$ such that the following hold: the singular measure $\nu^{s}$ defined in $\$ 2.7$ is supported on $N$, for every $y \notin N$ and every $C \in \mathscr{C}_{y}$ we have

$$
\int_{C} b \cdot \nabla \varphi+g \varphi d \mu_{y}=0 \quad \forall \varphi \in C_{c}^{\infty}\left(\mathbb{R}^{2}\right),
$$

and setting $F:=f^{-1}(N) \cup\left(\mathbb{R}^{2} \backslash E^{*}\right)$ we have

$$
\int_{F} b \cdot \nabla \varphi+g \varphi d x=0 \quad \forall \varphi \in C_{c}^{\infty}\left(\mathbb{R}^{2}\right) .
$$

Since $F$ is contained (up to a negligible subset) in the critical set $S$, we have that $b=0$ a.e. on $F$, and therefore (6.4) implies that

$$
g=0 \quad \text { a.e. in } F \text {. }
$$

Moreover, choosing a parametrization $\gamma$ of $C$ as in $\$ 4.2$ and recalling that the measure $\lambda$ which appears there is null because of the weak Sard property (Lemma 2.14), we can rewrite (6.3) as

$$
\int_{0}^{L} \tilde{a} \dot{\varphi}+\tilde{g} \varphi d s=0 \quad \forall \varphi \in C^{\infty}\left([0, L]^{*}\right),
$$


where we have set $\tilde{a}(s):=a(\gamma(s))$ and $\tilde{g}(s):=g(\gamma(s))$. This means that $\tilde{g}$ is the distributional derivative of $\tilde{a}$.

Now, the assumption $b=0$ a.e. on $A$ implies that $\tilde{a}=0$ a.e. on $\gamma^{-1}(A)$, and therefore the strong locality of derivatives of Sobolev functions yields $\tilde{g}=0$ a.e. in $\gamma^{-1}(A)$, that is,

$$
g=0 \quad \mu_{y} \text {-a.e. in } A \cap C .
$$

Since (6.7) holds for every $C \in \mathscr{C}_{y}$ and for $\nu$-a.e. $y$, the disintegration formula for the Lebesgue measure (cf. \$2.7) yields

$$
g=0 \quad \text { a.e. in } A \backslash F \text {, }
$$

and together with 6.5) gives that $g=0$ a.e. in $A$, which concludes the proof of statement (i).

We now prove statement (ii). Since $f$ does not satisfy the weak Sard property, there exists a set $G$ in $\mathbb{R}$ with positive measure such that for every $y \in G$ there exists $C \in \mathscr{C}_{y}$ for which the measure $\lambda$ is nontrivial (recall Lemma 2.14).

For every such $C$, consider the usual parametrization $\gamma$ and choose two bounded functions $\tilde{a}$ and $\tilde{g}$ on $[0, L]^{*}$ so that

$$
\partial_{s} \tilde{a}=\tilde{g}(1+\lambda)
$$

in the sense of distributions on $[0, L]^{*}$, and $\tilde{g}$ is not $\lambda$-a.e. null.

Now define the functions $g$ and $a$ on $\mathbb{R}^{2}$ so that $g=a=0$ out of the union of all $C$ given above, and $a(\gamma(s))=\tilde{a}(s)$ and $g(\gamma(s))=\tilde{g}(s)$ for $(\mathscr{L}+\lambda)$-a.e. $s$ and every $C$ given above.

It can be verified that $g$ is the distributional divergence of $b:=a \nabla^{\perp} f$, and the restriction of $g$ to the singular set $S$ is not a.e. null. Hence implication (6.2) fails for $A:=S$.

\section{Appendix: a measurable Selection lemma}

In this appendix we prove a measurable selection lemma used in the proof of Theorem 3.10. For the notation we refer to Section 2. Given $m, T>0$, we denote by $\mathscr{C}$ the set of all couples $(y, \eta)$ such that $y \in \mathbb{R}^{d-k}$ and $\eta$ is a real-valued measure on $[0, T) \times \mathbb{R}^{d}$ of the form

$$
\eta=u \cdot \mathscr{L}^{1} \otimes \mu_{y},
$$

where $u:[0, T) \times E_{y} \rightarrow \mathbb{R}$ satisfies $|u| \leq m$ everywhere and solves $(3.2)$ on $E_{y}$, or equivalently $\eta$ is a measure solution of $(3.2)$ in the sense of cf. 33.5 .

We denote by $\mathscr{M}$ the Banach space of real-valued measures on $[0, T) \times \mathbb{R}^{d}$. In the measure theoretic issues that will be considered below, the word "Borel" in relation to $\mathscr{M}$ refers to the $\sigma$-algebra generated by the weak* topology of $\mathscr{M}$ (that is, the one induced by the standard duality with the space of continuous functions on $[0, T) \times \mathbb{R}^{d}$ vanishing at infinity). The key point is that the restriction of the weak* topology to any closed ball of $\mathscr{M}$ is compact and metrizable, and therefore Polish (separable and completely metrizable). 
7.1. Proposition. The set $\mathscr{C}$ defined above is a Borel subset of $\mathbb{R}^{d-k} \times \mathscr{M}$.

Proof. Step 1. We rewrite $\mathscr{C}$ as the set of all $(y, \eta)$ such that

$$
|\eta|(E) \leq m \mathscr{L}^{1} \otimes \mu_{y}(E) \quad \forall E \subset[0, T) \times \mathbb{R}^{d},
$$

where $|\eta|$ denotes the variation of a real-valued measure $\eta$, and

$$
\int \partial_{t} \varphi+b \cdot \nabla \varphi d \eta=0 \quad \forall \varphi \in C_{c}^{\infty}\left([0, T) \times \mathbb{R}^{d}\right) .
$$

Indeed (7.1) means that the measure $\eta$ is absolutely continuous with respect to $\mathscr{L}^{1} \otimes \mu_{y}$ and that the density $u$ of the former measure with respect to the latter satisfies $|u| \leq m$ almost everywhere, while (7.2) means that $\eta$ is a measure solution of $(3.2)$.

Step 2: the set of all $(y, \eta)$ which satisfy $(7.1)$ is a Borel subset of $\mathbb{R}^{d-k} \times \mathscr{M}$. Note that (7.1) can be rewritten as

$$
\int \varphi d \eta \leq m \int|\varphi| d t d \mu_{y} \quad \forall \varphi \in C_{0}\left([0, T) \times \mathbb{R}^{d}\right) .
$$

Moreover it suffices that the inequality in $(7.3)$ is satisfied for all $\varphi \in \mathscr{D}$, where $\mathscr{D}$ is a given countable dense subset of $C_{c}\left([0, T) \times \mathbb{R}^{d}\right)$. In other words (7.3), and therefore also (7.1), can be rewritten as $\Lambda_{\varphi}(y, \eta) \leq 0$ for all $\varphi \in \mathscr{D}$, where

$$
\Lambda_{\varphi}(y, \eta):=\int \varphi d \eta-m \int|\varphi| d t d \mu_{y} .
$$

Now, the first integral in 7.4 is a continuous function of $\eta$ (by the definition of the topology on $\mathscr{M}$ ) while the second integral is a Borel function of $y$ (because the family $\left\{\mu_{y}\right\}$ is Borel, cf. $\$ 2.2$ and $\$ 2.3$, and therefore $\Lambda_{\varphi}$ is a Borel function on $\mathbb{R}^{d-k} \times \mathscr{M}$.

Hence the set of all $(y, \eta)$ satisfying $\Lambda_{\varphi}(y, \eta) \leq 0$ is Borel for every given $\varphi$, and so is the set of all $(y, \eta)$ satisfying $\Lambda_{\varphi}(y, \eta) \leq 0$ for all $\varphi \in \mathscr{D}$.

Step 3: the set of all $(y, \eta)$ which satisfy $(7.2)$ is a Borel subset of $\mathbb{R}^{d-k} \times \mathscr{M}$. Note that in $(7.2)$ we can equivalently require that the equality holds just for the test function $\varphi \in \mathscr{D}^{\prime}$, where $\mathscr{D}^{\prime}$ is a given countable dense subset of $C_{c}^{\infty}\left([0, T) \times \mathbb{R}^{d}\right)$. Therefore, arguing as in Step 2, we only need to show that for every $\varphi \in C_{c}^{\infty}\left([0, T) \times \mathbb{R}^{d}\right)$ the integral at the left-hand side of $(7.2)$ is a Borel function of $\eta$.

We actually prove that for every bounded Borel function $a:[0, T) \times \mathbb{R}^{d} \rightarrow \mathbb{R}$ the integral $\int a d \eta$ is a Borel function of $\eta$.

Let indeed $\mathscr{F}$ be the class of all bounded Borel real functions $a$ on $[0, T) \times \mathbb{R}^{d}$ such that this is true. One easily checks the following:

(i) $\mathscr{F}$ is a vector space;

(ii) if $f$ is the pointwise limit of an (increasing) sequence of uniformly bounded functions in $\mathscr{F}$, then $f$ belongs to $\mathscr{F}$;

(iii) $\mathscr{F}$ contains all functions in $C_{0}\left([0, T) \times \mathbb{R}^{d}\right)$ and therefore also all bounded continuous functions on $[0, T) \times \mathbb{R}^{d}$ by statement (ii). 
Then the functional version of the monotone class theorem (see for instance [12, Chapter I, Theorem 21]) implies that $\mathscr{F}$ contains all bounded Borel functions.

7.2. Corollary. Let $\mathscr{C}^{*}$ be the set of all $(y, \eta) \in \mathscr{C}$ with $\eta \neq 0$, and let $G$ be the projection of $\mathscr{C}^{*}$ on $\mathbb{R}^{d-k}$. Then $G$ is Lebesgue measurable, and there exist a Borel set $G^{\prime} \subset G$ such that $\mathscr{L}^{d-k}\left(G \backslash G^{\prime}\right)=0$ and a Borel family $\left\{\eta_{y}: y \in G^{\prime}\right\}$ such that $\left(y, \eta_{y}\right) \in \mathscr{C}^{*}$ for every $y \in G^{\prime}$.

Proof. Since $\mathscr{C}$ is a Borel set in $\mathbb{R}^{d-k} \times \mathscr{M}$, and so is (obviously) the set $\mathscr{I}$ of all couples $(y, 0)$ with $y \in \mathbb{R}^{d-k}$, then also $\mathscr{C}^{*}=\mathscr{C} \backslash \mathscr{I}$ is Borel.

In particular $\mathscr{C}^{*}$ belongs to the product $\sigma$-algebra generated by the $\sigma$-algebra of Lebesgue measurable sets on $\mathbb{R}^{d-k}$ times the Borel $\sigma$-algebra on $\mathscr{M}$. Therefore, by von Neumann's selection theorem (cf. [22, Corollary 5.5.8]), the projection $G$ is Lebesgue measurable, and there exists a Lebesgue measurable map that to every $y \in G$ associates $\eta_{y}$ such that $\left(y, \eta_{y}\right) \in \mathscr{C}^{*}$.

To conclude, we choose a Borel subset $G^{\prime}$ of $G$ such that the restriction of this selection map to $G^{\prime}$ is Borel, and $\mathscr{L}^{d-k}\left(G \backslash G^{\prime}\right)=0$.

\section{REFERENCES}

[1] M. Aizenman: On vector fields as generators of flows: a counterexample to Nelson's conjecture. Ann. Math., 107 (1978), 287-296

[2] G. Alberti, S. Bianchini, G. Crippa: Structure of level sets and Sard-type properties of Lipschitz maps. Preprint, 2010. Available at http://cvgmt.sns.it/

[3] G. Alberti, S. Bianchini, G. Crippa: On the $L^{p}$-differentiability of certain classes of functions. Preprint, 2011. Available at http://cvgmt.sns.it/

[4] L. Ambrosio: Transport equation and Cauchy problem for $B V$ vector fields. Invent. Math., 158 (2004), 227-260

[5] L. Ambrosio, F. Bouchut, C. De Lellis: Well-posedness for a class of hyperbolic systems of conservation laws in several space dimensions. Comm. Partial Differential Equations, 29 (2004), 1635-1651

[6] L. Ambrosio, G. Crippa: Existence, uniqueness, stability and differentiability properties of the flow associated to weakly differentiable vector fields. Transport equations and multi-D hyperbolic conservation laws, pp. 3-57. Lect. Notes Unione Mat. Ital., vol. 5, Springer, Berlin, 2008.

[7] F. Bouchut, L. Desvillettes: On two-dimensional Hamiltonian transport equations with continuous coefficients. Differential Integral Equations, 14 (2001), 1015-1024

[8] F. Colombini, G. Crippa, J. Rauch: A note on two-dimensional transport with bounded divergence. Comm. Partial Differential Equations, 31 (2006), 1109-1115

[9] F. Colombini, T. Luo, J. Rauch: Uniqueness and nonuniqueness for nonsmooth divergence-free transport. Séminaire Équations aux Dérivées Partielles, Exp. No. XXII. École Polytech., Palaiseau, 2003

[10] F. Colombini, J. Rauch: Uniqueness in the Cauchy problem for transport in $\mathbb{R}^{2}$ and $\mathbb{R}^{1+2}$. J. Differential Equations, 211 (2005), 162-167

[11] N. Depauw: Non-unicité du transport par un champ de vecteurs presque $B V$. Séminaire Équations aux Dérivées Partielles, Exp. No. XIX. École Polytech., Palaiseau, 2003 
[12] C. Dellacherie, P.-A. Meyer: Probabilities and potential. North-Holland Mathematics Studies, 29. North-Holland, Amsterdam-New York, 1978

[13] R.J. DiPerna, P.-L. Lions: Ordinary differential equations, transport theory and Sobolev spaces. Invent. Math., 98 (1989), 511-547

[14] R.J. DiPerna, P.-L. Lions: On the Cauchy problem for Boltzmann equations: global existence and weak stability. Ann. of Math. (2), 130 (1989), 321-366

[15] R.J. DiPerna, P.-L. Lions: Global weak solutions of Vlasov-Maxwell systems. Comm. Pure Appl. Math., 42 (1989), 729-757

[16] H. FEDERER: Geometric measure theory. Grundlehren der mathematischen Wissenschaften, 153. Springer-Verlag, New York 1969. Reprinted in the series Classics in Mathematics. Springer, Berlin-Heidelberg 1996.

[17] M. Hauray: On two-dimensional Hamiltonian transport equations with $L_{\mathrm{loc}}^{p}$ coefficients. Ann. Inst. H. Poincaré Anal. Non Linéaire, 20 (2003), 625-644

[18] M.W. Hinsch: Differential topology. Graduate Texts in Mathematics, 33. SpringerVerlag, New York 1976

[19] S.G. Krantz, H.R. Parks: Geometric integration theory. Cornerstones. Birkhäuser Boston, Boston, 2008

[20] A. SARD: The measure of the critical values of differentiable maps. Bull. Am. Math. Soc., 48 (1942), 883-890

[21] L. Simon: Lectures on geometric measure theory. Proceedings of the Centre for Mathematical Analysis, 3. Australian National University, Canberra, 1983

[22] S.M. Srivastava: A course on Borel sets. Graduate Texts in Mathematics, 180. Springer, New York, 1998

[23] W.P. Ziemer: Weakly differentiable functions, Sobolev spaces and functions of bounded variation. Graduate Texts in Mathematics, 120. Springer-Verlag, New York, 1989

G.A.

Dipartimento di Matematica, Università di Pisa, largo Pontecorvo 5, I-56127 Pisa, Italy

e-mail: galberti1@dm.unipi.it

S.B.

S.I.S.S.A., via Bonomea 265, I-34136 Trieste, Italy

e-mail: bianchin@sissa.it

G.C.

Departement Mathematik und Informatik, Universität Basel, Rheinsprung 21, CH-4051 Basel, Switzerland

e-mail: gianluca.crippa@unibas.ch 